\title{
The risk of drug resistance during long-acting antimicrobial therapy
}

\author{
Anjalika Nande ${ }^{1,2}$ and Alison L. Hill*1,2 \\ ${ }^{1}$ Program for Evolutionary Dynamics, Harvard University, Cambridge, MA 02138 \\ ${ }^{2}$ Institute for Computational Medicine, Johns Hopkins University, Baltimore, MD 21218
}

\begin{abstract}
The emergence of drug resistance during antimicrobial therapy is a major global health problem, especially for chronic infections like HIV, hepatitis B and C, and TB. Sub-optimal adherence to long-term treatment is an important contributor to resistance risk. New long-acting drugs are being developed for weekly, monthly, or less frequent dosing to improve adherence, but may lead to long-term exposure to intermediate drug levels. In this study we analyze the effect of dosing frequency on the risk of resistance evolving in the presence of time-varying drug levels. We find that long-acting therapies can increase, decrease, or have little effect on resistance, depending on the source (pre-existing or de novo) and degree (full or partial) of resistance, and whether the drug is absorbed - as well as cleared - more slowly. For long-acting therapies with slow drug clearance but rapid absorption, and for partially-resistant strains, longer dosing intervals tend to reduce resistance risks even if they don't improve adherence, and adherence improvements amplify these effects. In other scenarios, long-acting therapies are more susceptible to resistance and must substantially improve adherence to overcome this deficit. If subpopulations of microbes persist and can reactivate during suboptimal treatment, longer-acting therapies may substantially increase the risk of resistance. Our results show that drug kinetics affect selection for resistance in a complicated manner, and that pathogen-specific models are needed to evaluate the benefit of new long-acting therapies.
\end{abstract}

*Corresponding author: alhill@jhu.edu 


\section{Introduction}

In recent decades, highly effective drugs have helped reduce morbidity and mortality of chronic viral infections, like those caused by the human immunodeficiency virus (HIV) [1] and hepatitis B (HBV) [2] and $\mathrm{C}(\mathrm{HCV})[3]$ viruses. However, effective treatments can become ineffective due to the emergence of drug resistant strains [4]. Drug resistance can evolve rapidly within individual hosts due to the large population sizes [5] and high replication and mutation rates of many viruses [6]. Similar problems complicate treatment for chronic bacterial infections like tuberculosis (TB) [7]. Chronic infections require long treatment courses (months - years), increasing the opportunity for these pathogens to adapt.

An effective treatment can also fail due to non-adherence (missed doses). Typical rates of adherence for medications prescribed over a long period of time have been found to be between $50-75 \%$ [8]. In some cases this may be far less than what is needed for a treatment to be effective. For some antiretroviral therapies for HIV, studies have estimated that patients need near perfect ( $>95 \%)$ adherence for successful viral suppression [9], while for HBV adherence levels $>80 \%$ have been associated with an approximately $90 \%$ reduction in the rate of treatment failure [10].

Long-acting drugs are being developed as one of the ways to help address the problem of imperfect adherence [11]. For example, a two-drug injectable treatment regimen is being developed for HIV using cabotegravir and rilpivirine, which are formulated to have a multi-week half-life. It would be administered only once every 4 or 8 weeks as opposed to current daily dosing [12-16]. Large investments are being made in developing long acting treatments for $\mathrm{HCV}$ and TB, and prophylaxis for TB and malaria [17, 18], with some success already in animal models [19-21]. Long-acting lipoglycopeptide antibiotics are already available to treat bacterial skin infections [22-24]. Monoclonal antibodies are an emerging treatment for infectious diseases which can be engineered to have long half-lives [25]. While long-acting therapy is likely to increase overall patient adherence, we do not yet know how this would affect resistance. Many studies have shown that sub-optimal adherence to daily pills contributes to resistance [26-33], but it is also possible that the long-term exposure to intermediate drug levels between doses of a long-acting drugs could facilitate the evolution of resistance [34,35]. The goal of this study is to examine the role of drug dosing kinetics on the risk of drug resistance.

Drug resistance can arise from two sources: mutants that exist prior to treatment initiation or those that are produced during treatment $[36,37]$. The relative contribution of these sources towards drug resistance is very difficult to separate experimentally, but has been thoroughly investigated in a generalized model of intra-host viral dynamics for a completely resistant mutant in the presence of constant drug efficacy [37]. However, recent work on evolution in fluctuating environments [38] shows that the fate of mutants that are under time-dependent selection pressures can't necessarily be predicted by the time-averaged selective effect alone; suggesting the effect of drug kinetics should be important. Numerous studies have integrated pharmacokinetics into mathematical models of infection dynamics [39-51], and some have shown that the likelihood of generating and selecting for drug resistance depends on fluctuating drug levels. However, no studies have systematically studied the impact of changing a drug profile, as will occur with the reformulation of drugs into long-acting therapies.

In this study we expand previous models to analyze the effect of drug kinetics on the evolution of resistance, focusing specifically on the periodicity of drug dosing. We incorporate competition between wild-type and resistant strains, the fitness costs and benefits of resistance, pharmacologically-relevant drug kinetics, and treatment adherence. We consider pre-existing and rescue (de novo) mutations as well as mutations arising on account of reactivation of latent infection.

We find that drug kinetics have a complicated effect on the selection and competition for the resistant strain. Depending upon the source of resistance (pre-existing or de novo), degree of resistance of the mutant strain (partial or complete), and the rates of drug absorption and clearance, long-acting therapies can increase, decrease or have no effect on the establishment of resistance. These findings accentuate the need to incorporate pharmacokinetic, pharmacodynamic, and evolutionary considerations into models used to guide drug development and implementation for rapidly evolving pathogens. 


\section{Model}

To understand the impact of drug kinetics on the evolution of resistance, we used a stochastic model of viral dynamics within individual hosts [52]. This model (Figure 1A) describes the interactions between target (uninfected) cells, drug-susceptible wild-type virus (WT), and a drug-resistant virus strain, in the presence of treatment $[37,53]$. While this model was designed for chronic viral infections, our results are generalizable to other infections with density-dependent growth and direct-acting, infection-blocking therapeutics [43,47]. For now, we assume that the resistant mutant is generated from the WT by a single point mutation. We discuss the implications of more complex mutational pathways in the last section. The full equations of the model are detailed in the Supplementary Methods and our code is open access on GitHub https://github.com/anjalikanande/drugkinetics-resistance.

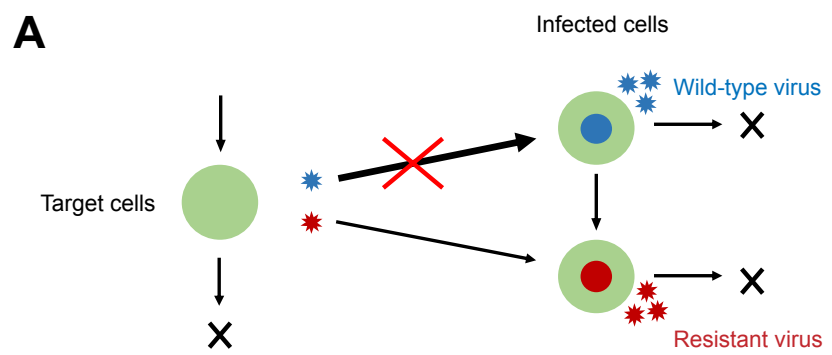

\section{Sinusoidal drug efficacy}

B

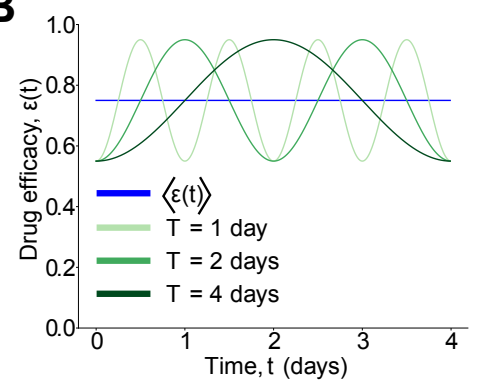

C

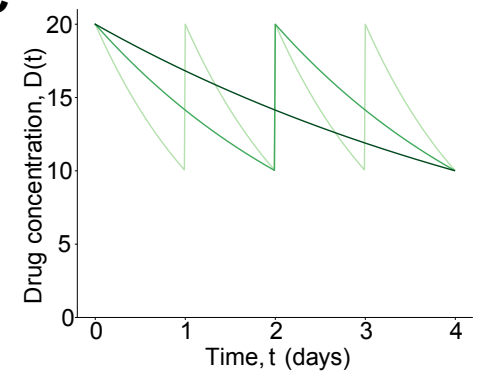

Pharmacologically-inspired drug efficacy

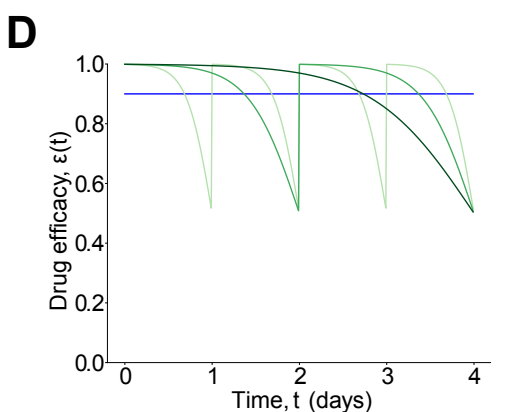

Figure 1: Model schematic and example time-dependent drug efficacy profiles A) A schematic of the viral dynamics model, consisting of uninfected target cells (green) and cells infected with either the wild-type (WT) virus (blue) or drugresistant virus (red). We assume that treatment blocks infection with the WT virus (red cross), while the resistant strain can still (at least partially) infect cells. The resistant virus is assumed to have a fitness cost, so in the absence of treatment, the WT virus is more infectious and dominates the population. The resistant strain can be produced via mutation from the WT. See Suppl. Methods for more details. B) Time-dependent drug efficacy under the sinusoidal efficacy model. The three green curves correspond to drug profiles with the same time-averaged efficacy (blue line) and maximum and minimum efficacy, but with different dosing periods, $T$ (darker curves correspond to longer T). C-D) Pharmacologically-inspired drug efficacy model. C) Drug concentration and D) drug efficacy as functions of time. Each green curve has the same time-averaged drug concentration but the dosing interval and half-life increase as the color darkens. Parameters values used for sinusoidal efficacy, $\epsilon_{\text {avg }}=0.75$, $A=0.20$ and for pharmacologically-inspired efficacy, $C_{\max }=20, I C_{50}=10, M=10, t_{h}=T$ and $\rho=1$. Drug concentrations are in arbitrary units.

Like most infection models, pathogen fitness in our model is encapsulated by the basic reproductive ratio, $R_{0}$, which is a composite of multiple individual parameters (Suppl. Methods). $R_{0}$ is defined as the average number of new infected cells produced in a single replication cycle by one infected cell in an otherwise susceptible population [52]. A viral strain $i$ can establish infection only if $R_{0}^{i}>1$. When there are multiple strains with $R_{0}^{i}>1$, competitive exclusion occurs and only the strain with highest $R_{0}$ can sustain high-level infection. We assume that resistance is accompanied by some fitness cost, $0 \leq s<1$. Consequently, in the absence of treatment, $R_{0}^{w}>R_{0}^{r}$, and infection is predominantly with the wild-type strain. The resistant 
strain is maintained in the population at low levels by a mutation-selection balance (Figure $\mathbf{S 1}$ ), which may lead to pre-existing resistance.

We assume that drug treatment reduces the rate of infection by a factor $1-\epsilon$, where $0<\epsilon<1$ is the drug efficacy. The effect of the drug on the resistant strain is less than that on the wild-type strain $\left(\epsilon_{r}<\epsilon_{w}\right)$. We are interested in a regime where before treatment, the wild-type has established infection $\left(R_{0}^{w}>1\right)$, but during treatment, the wild-type is suppressed $\left(R_{0}^{w}\left(1-\epsilon_{w}\right)<1\right)$ whereas the resistant strain is not $\left(R_{r}\left(1-\epsilon_{r}\right)>1\right)$. Since drug concentrations are time-dependent, we allow drug efficacy to depend on time as well, $\epsilon(t)$.

Throughout the paper, we consider two example drug efficacy profiles. The first is a simple sinusoidal function (Figure 1B),

$$
\epsilon(t)=\epsilon_{\mathrm{avg}}-A \cos \left(\frac{2 \pi t}{T}\right)
$$

where, $\epsilon_{\text {avg }}$ is the time-averaged drug efficacy, $A$ is difference between the average and peak or trough efficacy (amplitude), and $T$ is the dosing interval (period). Although a heuristic function, we interpret this model as follows: When a dose is taken, the efficacy starts at the lowest level, and then increases as the drug is absorbed in the body and eventually reaches a maximum level. After this time, drug clearance dominants, lowering the concentration until the next dose is taken and the cycle repeats. The average drug efficacy may be different for WT and resistant strains.

Secondly, we consider a more pharmacologically-inspired model where the efficacy is related to the drug concentration by a Hill dose-response curve (Figures 1C,D). We assume that drug absorption is rapid compared to drug clearance (as is the case for many drugs), and approximate the drug concentration $D(t)$ as an instantaneous jump to the peak level followed by exponential decay. Together, this gives

$$
\begin{aligned}
D(t) & =C_{\max } 2^{-\frac{t}{t_{h}}} \\
\epsilon_{i}(t) & =1-\frac{1}{1+\left(\frac{D(t)}{\rho_{i} I C_{50}}\right)^{M}}
\end{aligned}
$$

The maximum drug concentration is $C_{\max }$ and the half-life is $t_{h}$. The efficacy of the drug against strain $i$ at time $t$ is $\epsilon_{i}(t)$, where, $I C_{50}$ is the drug concentration that achieves half-maximal efficacy, $M$ quantifies the steepness of the dose-response curve, and $\rho_{i}$ corresponds to the extent of drug resistance for a particular strain $\left(\rho_{i}=1\right.$ for $\mathrm{WT}$ and $\rho_{i}>1$ if resistant).

With each of these models, we systematically varied the dosing interval while keeping the average efficacy the same (as well as the peak and trough efficacy). This is meant to mimic the scenario under which longacting therapies are being developed: we assume that if the drug decay can be slowed down by some amount, the dosing interval is extended by the same amount. In the sinusoidal drug model, we vary the drug dosing period, $T$. In the pharmacological model, we varied the drug half-life, $t_{h}$.

Conceptually, there are two possible ways drug resistance can emerge to therapy [36,37]. Drug resistant strains may pre-exist at the start of therapy, since they are continually produced by the WT strain and exist at mutation-selection balance even in the absence of therapy. Once the WT infection is suppressed by therapy, the resistant strain has less competition for target cells (the "resource"), and can potentially establish its own infection. However, establishment is not guaranteed: the population of pre-existing resistant mutants can be very small and subject to stochastic extinction. Resistant infection can also be established by mutants produced by residual WT replication despite therapy, either at the start of treatment or before treatment has cleared the initially present WT population (referred to as "rescue mutants" following [37], a term borrowed from population genetics literature [54,55]), or later on in the treatment course due to reactivation of latent infected cells (only for certain infections). These mutants are also subject to stochastic extinction. In the following sections, we calculate the establishment probability of resistant infection due to these sources and analyze the effect of the drug dosing interval on each of them. 


\section{Results}

\section{Resistant mutants existing prior to treatment}

We first examined the impact of the dosing interval on the evolution of resistance from pre-existing mutants. Extending the approach of Alexander \& Bonhoeffer [37], we calculated the establishment probability $p_{\text {est }}$ for a single pre-existing resistant mutant in the presence of treatment with time-dependent drug levels (Suppl. Methods). We first examined the case of a fully-resistant mutant (a strain that is not impacted at all by the drug level, purple curves in Figures 2B,E). Under the sinusoidal model of drug efficacy (slow drug absorption), longer dosing intervals (i.e. long-acting therapy) always lead to lower probabilities of resistant mutants establishing. In contrast, under the pharmacological model (fast drug absorption), increasing the dosing period makes it more likely the resistant strain will establish. However in either case, the differences in $p_{\text {est }}$ were minimal.

We next looked at the case where the mutant is only partially resistant (green curves in Figures 2B,E). Overall the establishment probability was lower and more sensitive to the drug dosing interval. When drug efficacy fluctuated sinusoidally between doses, longer dosing intervals only led to lower establishment probability up to a certain maximal dose period ( $\sim$ monthly for the parameters we used), and then further increasing the period leads to even higher establishment probabilities than daily dosing. When drug efficacy followed a pharmacological model, increased dose intervals decreased establishment probability. Therefore, the effect of increasing dosing intervals on the risk of resistance due to pre-existing mutants seems to depend strongly on both the degree of resistance and on the details of the drug kinetic curve.

We find that there are two limiting cases: when dosing is frequent the establishment probability depends upon the time-averaged drug efficacy, whereas in the limit of very infrequent dosing it depends upon the initial drug efficacy $(\epsilon(t=0))$. The existence of these limiting cases and the effect of dosing intervals can be explained by the time-scales and selection forces in the system. There exists an initial time-frame after treatment is initiated that is most important for pre-existing mutants to establish a resistant infection. This time-frame exists because a) mutant virus and the cells it infects have a finite lifespan, and b) competition with the WT for target cells (or more generally, any limited resource). Although the resistant strain has a higher fitness than the WT strain once therapy begins, initially target cells are suppressed such that $R_{r}<1$ and hence $p_{\text {est }}=0$. Target cell numbers start increasing once treatment starts since WT replication is suppressed. The resistant strain can establish infection only when target cells have increased sufficiently such that $R_{r}>1$. This leads to the important time-scale in the system which we refer to as the Establishment Time-Frame (ETF). If the lineage of a resistant strain has managed to survive through the ETF, then it is almost guaranteed to establish infection. This can be seen by tracking how quickly the fraction of preexisting mutants approaches the establishment probability after the onset of treatment (Figure S2). The establishment probability therefore depends mainly upon the drug kinetics during this time.

This theory explains the existence of two limiting cases for very short or very long dosing intervals. When drug dosing is frequent, the drug undergoes many cycles during the ETF and the effect of the drug is well-approximated by a constant drug efficacy at the time-averaged value (see blue stars in Figures 2B,E). In the limit of very infrequent dosing (very long-acting drugs), the drug stays near its initial concentration for the duration of the ETF. Consequently, the establishment probability approaches that of a constant drug efficacy at the initial drug level $(\epsilon(t=0)$, orange stars in Figures 2B,E). One important difference between the sinusoidal vs the pharmacological drug model, which explains the different effects of long-acting therapy in the two cases, is the value of the drug concentration early on. In the sinusoidal model, drug absorption is assumed to be slow and so drug levels are assumed to be near their minimum during the ETF for extended-release therapy. In the pharmacological model, drug levels are assumed to reach their peak quickly, and so the resistant mutant experiences peak efficacy during the ETF under long-acting therapy.

Two opposing selection forces also contribute to the relationship between the drug efficacy and the establishment probability. Suppression of the WT by the drug helps the growth of the resistant strain indirectly by reducing competition for available target cells. But if the mutant strain is not completely resistant, then suppression of the resistant strain by the drug directly reduces its fitness. Consequently, higher drug efficacy can both help and hurt the resistant strain. When the suppression of the resistant strain by the drug is the dominant selection force, higher drug efficacy during the ETF lowers the establishment probability (partial resistance in Figures 2B,E). On the other hand, higher drug efficacy during the ETF 
medRxiv preprint doi: https://doi.org/10.1101/2021.07.10.21260044; this version posted July 14, 2021. The copyright holder for this preprint (which was not certified by peer review) is the author/funder, who has granted medRxiv a license to display the preprint in perpetuity.

It is made available under a CC-BY 4.0 International license.

\section{Sinusoidal drug efficacy}

A

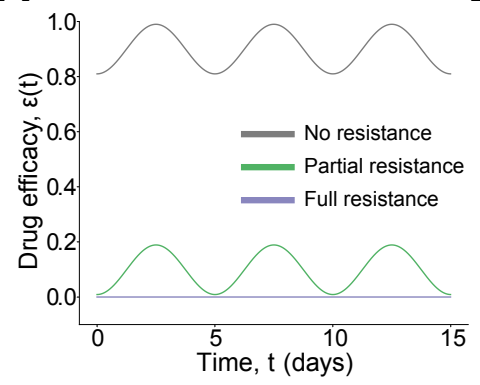

B

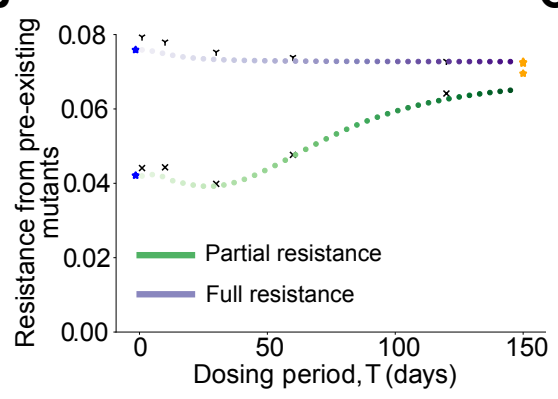

C

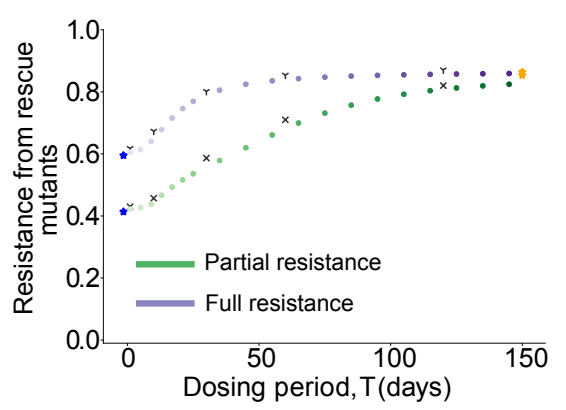

\section{Pharmacologically-inspired drug efficacy}
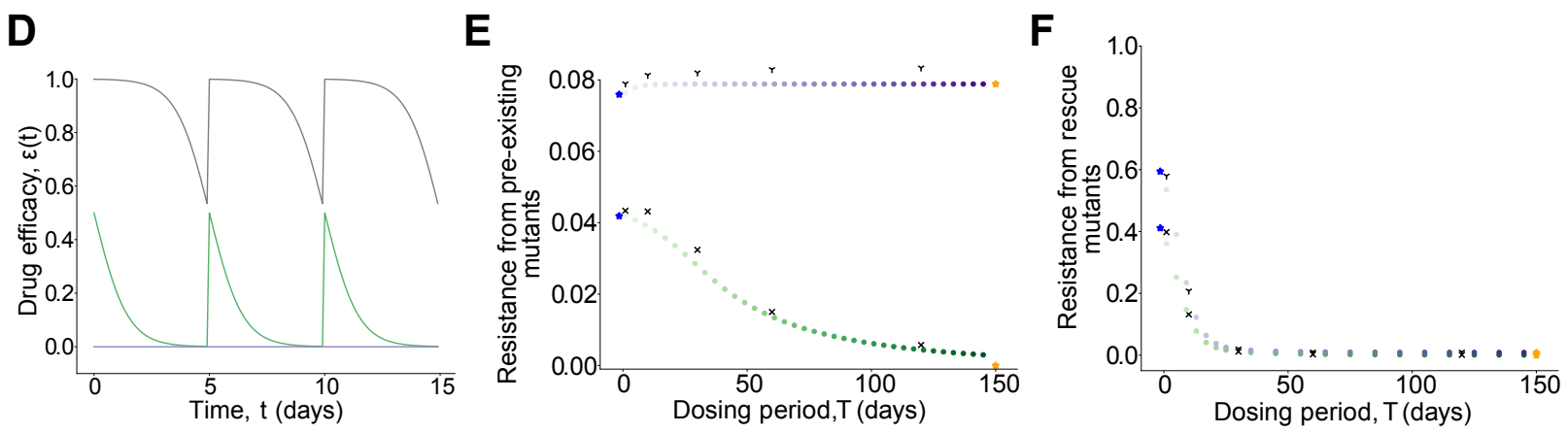

Figure 2: Effect of drug dosing intervals on the establishment of resistance due to pre-existing and rescue mutants. Top row: Results for the sinusoidal drug efficacy model. Bottom row: Results for the pharmacologically-inspired drug efficacy model. A) and D) Drug efficacy as a function of time for the WT and resistant strain. B) and E) Establishment probability for one pre-existing resistant mutant as a function of the dosing interval. C) and F) Probability that at least one rescue mutant is produced as a function of the dosing interval. Each dot represents the establishment or rescue probability in the presence of an oscillatory efficacy with a fixed period. The colors go from light to dark with increasing dosing intervals keeping the time-averaged efficacy, $\epsilon_{\text {avg }}$ constant. In each case, the establishment and rescue probabilities were computed for mutants unaffected by the drug $\epsilon_{r}=0$ (purple) and affected by the drug $\epsilon_{r} \neq 0$ (green). The starred points correspond to the establishment (rescue) probability for constant efficacies, $\epsilon_{\mathrm{avg}}=\langle\epsilon(t)\rangle$ (blue star) and $\epsilon=\epsilon(t=0)$ (orange star). The black marks overlaid on each of the curves are results from stochastic simulations of the whole model, see Suppl. Methods for details. Parameter values used for the drug kinetics in A-C), $\epsilon_{\mathrm{avg}, \mathrm{w}}=0.9, A_{w}=0.09, \epsilon_{r}(t)=0$ (purple) and $\epsilon_{\mathrm{avg}, \mathrm{r}}=0.1, A_{r}=0.09$ (green) and in D-F), $C_{\max }=20, I C_{50}=10, M=10, \frac{t_{h}}{T}=1, \rho_{w}=1, \rho_{r}=\infty$ (purple) and $\rho_{r}=2$ (green). 
increases the establishment probability when competition with the WT is the dominant selection force (full resistance in Figures 2B,E). The effect of drug kinetics on the establishment probability therefore reflects the fitness of the resistant strain during the ETF. We investigate the dependence of model parameters on these results in the Supplement (Figures S3,S4, Suppl. Discussion).

To summarize, the effect of dosing intervals on the establishment of pre-existing resistant strains is minimal when the degree of resistance of the mutant strain is high. For a highly suppressive drug, longacting therapy tends to increase the risk of resistance due to pre-existing partially resistant mutants when the drug absorption is slow, and decreases the risk when drug absorption is fast. However, for a slow absorbing drug, there exists an intermediate regime where increasing the dosing interval leads to the reduction in the risk of resistance albeit by a small amount.

\section{Resistant mutants produced during treatment}

Next, we examined the effect of dosing intervals on the evolution of resistance due to mutants produced during treatment. The majority of the resistant mutants that are produced during treatment do not establish infection on account of stochastic extinction. As a result, we focus our analysis only on those resistant mutants that are produced during treatment and escape stochastic extinction by producing long lived descendants ("rescue mutants") . The rate at which rescue mutants are produced is therefore a combination of the rate of mutant production from residual WT replication and the probability of fixation (related to the strength of selection). We computed the probability that at least one rescue mutant is produced during treatment (see Suppl. Methods, [37]), and analyzed how it was affected by varying the drug dosing interval (Figures 2C,F).

We found that under the sinusoidal model (slow drug absorption), long-acting drugs increase the risk of resistance (Figures 2C). Whereas, under the pharmacologically-inspired model (fast drug absorption), longer acting drugs reduce the risk of resistance (Figures $\mathbf{2 F}$ ). These trends are independent of the degree of resistance of the mutant strain.

As in the case of pre-existence, there are two limiting cases corresponding to very frequent and infrequent dosing. When dosing is frequent, the rescue probability depends upon the time-averaged drug efficacy, whereas in the limit of very infrequent dosing it depends upon the initial drug efficacy. These results suggest that the rescue probability is also influenced by the early time dynamics and depends mainly upon the drug kinetics during the establishment time-frame (ETF).

The effect of dosing intervals and the trends seen in Figures $\mathbf{2 C , F}$ can be explained by the factors affecting the rescue probability. The risk of resistance depends upon the rate of production of rescue mutants which depends both on mutation and selection,

$$
\nu_{\text {resc }}(t)=\nu(t) p_{\text {est }}(t)
$$

where $\nu(t)$ is the rate at which resistant mutants are produced from residual WT infection and $p_{\text {est }}(t)$ is the establishment probability for such a mutant produced at time $t$. Treatment affects these factors in different ways. A stronger treatment is always associated with a lower $\nu(t)$ as there is less residual WT infection. On the other hand, a stronger treatment can be associated with a higher or lower $p_{\text {est }}(t)$ depending upon the degree of resistance of the mutant strain. This was the reason behind the opposite trends seen in the case of pre-existence (for example in Figure 2B). In the case of rescue mutants, however, the effect of varying the dosing interval (Figures $\mathbf{2} \mathbf{C , F}$ ) is independent of the degree of resistance of the mutant strain. A higher drug efficacy always corresponding to a lower rescue probability. This implies that the rate of mutant production $\nu(t)$ dominates selection $p_{\text {est }}(t)$. The effect of dosing intervals on the rescue probability is also dependent upon other model parameters and we investigate this dependence thoroughly in the Supplement (Figures S5,S6, Suppl. Discussion).

In summary, long-acting therapy increases the risk of resistance due to rescue mutations when drug absorption is slow, and decreases the risk of resistance when drug absorption is fast.

\section{Resistant mutants produced due to latency reactivation}

The infection model considered so far does not take into account persistent infected cell populations [56]. As a result, the infection tends to be cleared quickly as long as resistance doesn't emerge, which is why the early 
drug kinetics play an out-sized role in the risk of drug resistance. Persistence, however, is a characteristic of many chronic infections (e.g. HIV, HBV, TB, some types of malaria) $[7,57]$ that makes it difficult to design effective treatment strategies to completely clear the infection. It is also an important source of resistance. Reactivation events from this pool ("latent reservoir") of persistent infected cells can lead to the introduction of resistant mutants that establish infection. The rate at which resistant mutants are produced and go on to establish infection ("rescue" due to reactivation) is dependent upon the details of the drug kinetics and hence, we expect it to be influenced by the drug dosing intervals. With this in mind, we investigated how long-acting therapy affects the emergence of a resistant infection due to the reactivation of latent infected cells.

We modeled two ways in which resistant mutants can be introduced during a persistent infection (Suppl. Methods) - reactivation of a latently-infected cell that is already resistant (we assume that a fraction of the latent population given by mutation-selection balance is resistant), or reactivation and subsequent replication of WT infection that generates a resistant mutant (Figure 3A). Summing up both these possibilities, we calculated the average probability of rescue per latency reactivation event. This probability was calculated for reactivation events that occur after the initial period where the WT infection is being suppressed by treatment and target cells are being replenished to their pre-infection values (Suppl. Methods).
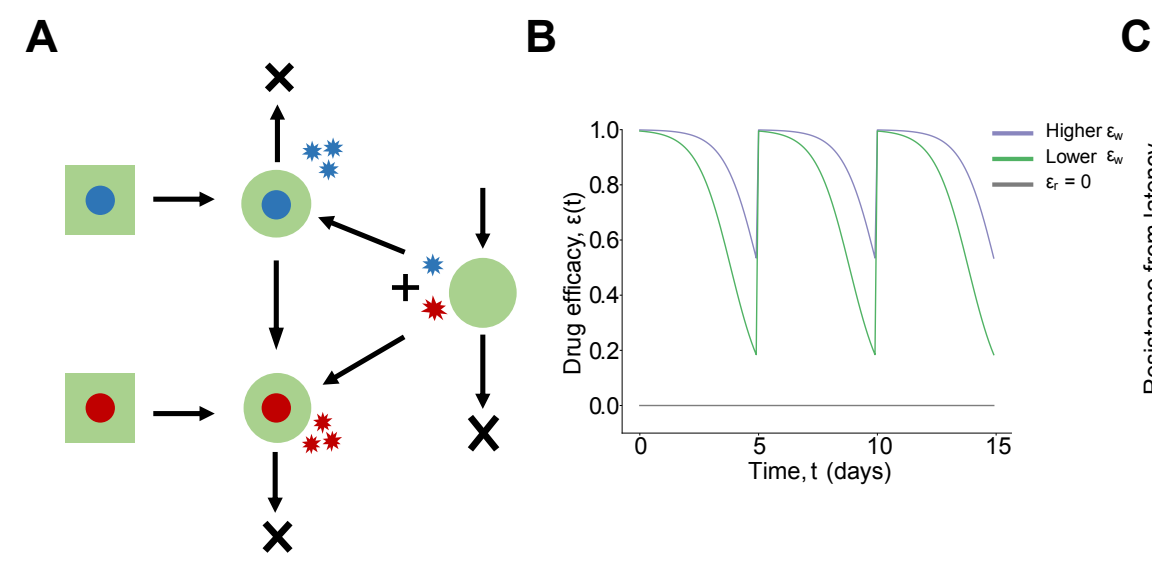

Figure 3: Effect of drug dosing intervals on the establishment of resistance due to latency reactivation. A) Schematic of the model showing the two ways in which resistant mutants can be produced as a result of the reactivation of latent infected cells (square). B) Drug efficacy as a function of time with two strengths of efficacies for the WT and fully resistant mutant, $\epsilon_{r}=0$. The purple curve corresponds to a high drug efficacy such that $R_{w}<1$ during all points of the drug cycle. $R_{w}>1$ for $\sim 23 \%$ of each period for the low efficacy drug cycle given by the green curve. C) Average probability of rescue per latency reactivation event as a function of the drug dosing interval. Each dot corresponds to the average probability in the presence of an oscillatory efficacy with a fixed dosing interval. The colors go from light to dark with increasing periodicity keeping the time-averaged efficacy, $\epsilon_{\text {avg }}$ constant. Black marks denote results of the stochastic simulation of the whole model. Parameter values used for the drug kinetics, $C_{\max }=20$ (purple curve), $C_{\max }=17$ (green curve), $I C_{50}=10, M=10, \frac{t_{h}}{T}=1$, $\rho_{w}=1, \rho_{r}=\infty$.

We found that depending upon the extent of WT suppression during the drug cycle, long-acting therapies are associated with the same or increased risk of resistance as compared to frequent dosing (Figure 3C). When the drug efficacy is high enough that the WT is suppressed $\left(R_{0}^{w}<1\right)$ for the entire the drug cycle, the average rate of rescue has no dependence upon the dosing interval (purple curve in Figure 3C). In the absence of WT replication, resistance can arise only via mutants that pre-exist in the latent reservoir. As the rate of reactivation is constant and independent of the drug kinetics, the risk of resistance in this case does not depend upon the drug dosing frequency. However, when the drug efficacy is lower and the WT can replicate during the times of lowest drug levels $\left(R_{w}>1\right)$, despite still being suppressed overall $\left(\left\langle R_{w}\right\rangle<1\right)$, the rate of rescue has a strong dependence upon the dosing intervals and increases for longer acting drugs (green curve in Figure 3C). The slow decay of longer acting drugs leads to more time in the non-suppressive part of the dosing period, which enables the WT to undergo multiple rounds of replication before eventually getting suppressed by the next drug dose, and increases the chance that a resistant mutant is produced and 
establishes infection. These results are irrespective of the degree of resistance of the mutant strain (Figure S7A) and the drug absorption rates (Figure S7B).

In summary, during a persistent infection, long-acting therapies are associated with an increased risk of resistance if there is sub-optimal WT suppression in the lowest parts of the drug cycle.

\section{Impact of non-adherence on resistance for different dosing intervals}

In the previous sections we have shown how drug kinetics influence the risk of resistance when there is perfect adherence to treatment. In reality, adherence to treatment is known to be sub-optimal, especially for chronic infections, and is associated with the risk of resistance for daily therapies. We therefore evaluated how imperfect adherence to therapy influences the relationship between drug dosing intervals and the risk of resistance. We assumed each scheduled dose was taken or missed randomly and independently with a probability given by the adherence level (Suppl. Methods) and analyzed the effect of dosing intervals on the probability of a resistant infection due to the different sources of mutations, for varying levels of adherence (Figure 4). While in reality low enough adherence levels can allow for treatment failure to occur without resistance, just due to rebound of the WT strain, we were not interested in this effect in this analysis and so ensured that the system stayed in the regime where the WT has a lower fitness than the resistant strain and is suppressed by treatment.

\section{Full resistance}

A

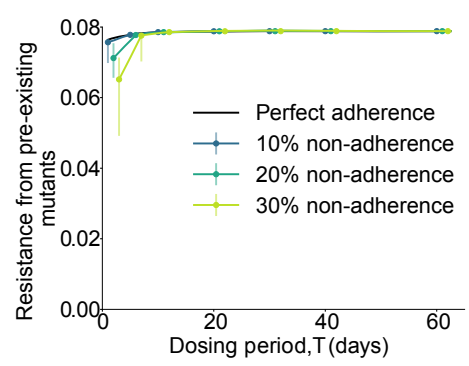

\section{Partial resistance}

D

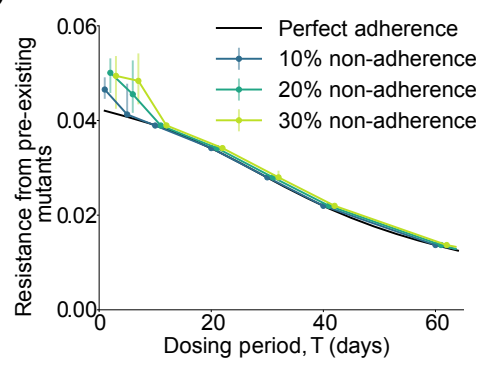

B

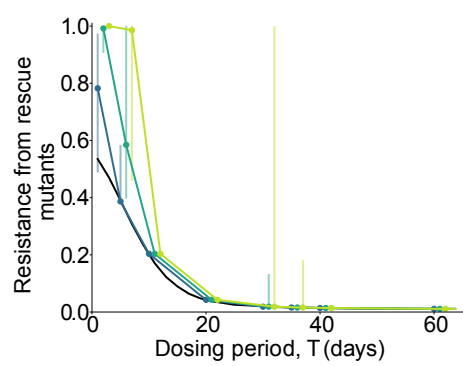

E

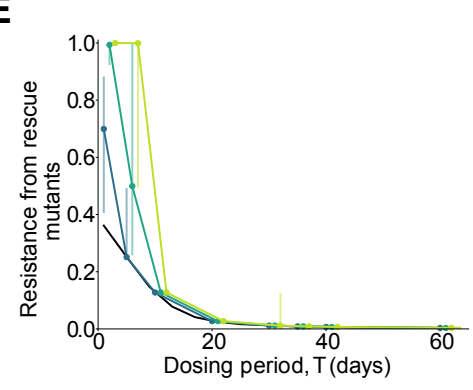

C

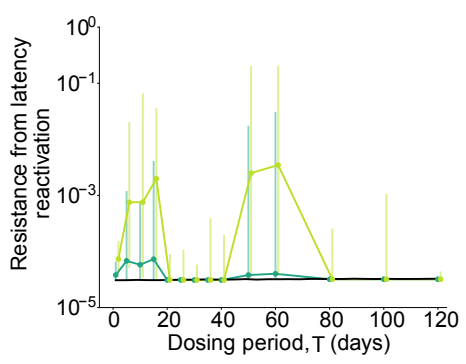

$\mathbf{F}$

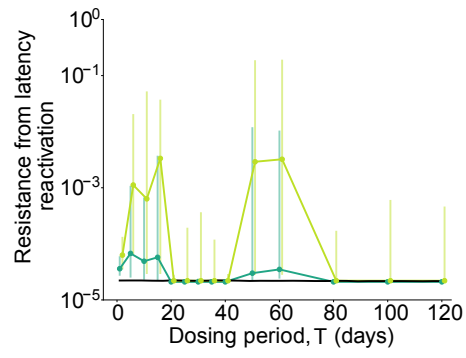

Figure 4: Effect of non-adherence on the establishment of resistance for different drug dosing intervals. Top row: Results for a fully resistant mutant $\left(\rho_{r}=\infty\right)$. Bottom row: Results for a partially resistant mutant $\left(\rho_{r}=2\right)$. A) and D) Establishment probability of pre-existing mutants. B) and E) Probability that at least one rescue mutant is produced. C) and F) Average probability of rescue per latency reactivation event. These results are for the pharmacologically-inspired drug efficacy model with the solid black line in each subplot corresponding to results for perfect adherence. Imperfect adherence results are medians of 100 iterations for pre-existence and rescue, and 500 iterations for latency. The error bars correspond to the interquartile range. X-axis positions have been offset for ease of visualization.

We found that for both pre-existing and rescue mutants, long-acting drugs were most robust to the effects of treatment non-adherence, with the same observed overall trends relating the risk of resistance to the frequency of dosing as for perfect adherence (Figures $\mathbf{4 A , B , D}$ and $\mathbf{E}$ ). Long-acting drugs are more robust in this case because the risk of resistance is mainly dependent upon drug kinetics during the establishment time-frame (ETF). The number of doses taken during the ETF decreases as the dosing interval increases, reducing the chance of missing a dose. As by definition the first dose is always taken, the risk of 
resistance is the same as that for perfect adherence when the dosing interval is equal to or larger than the ETF. These results are for the pharmacologically-inspired drug efficacy model and are independent of the specific model chosen for adherence (Figure S8).

It is worth noting that in agreement with previous modeling work [43,44], we found that non-adherence generally - but not always - promotes the emergence of resistance. In the case of fully resistant pre-existing mutants, lower adherence lowers the establishment probability (Figure 4A). Lower adherence corresponds to weaker WT suppression. As the resistant strain is unaffected by treatment, weak WT suppression increases competition for target cells, making it more difficult for the resistant strain to establish infection.

Depending upon the drug dosing period, imperfect adherence can significantly increase (by orders of magnitude) the risk of resistance during a persistent infection irrespective of the degree of resistance of the mutant strain (Figures $\mathbf{4 C , F}$ ). When drugs are very long-acting (drug dosing period larger than $\sim 2$ months), imperfect adherence doesn't play much of a role as in this case missing a dose leads to the rebound of the WT infection and doesn't favor the selection of the resistant strain. For shorter dosing periods however, the risk is predominantly increased apart from an intermediate regime. This trend is a combined effect of the rate at which resistant mutants are produced from the WT and competition between the two strains for uninfected cells once the resistant mutants have been produced. An increased risk corresponds to the situation where missing a dose leads to more WT replication which leads to a higher rate of mutant production but there is enough WT suppression once the mutant is produced such that it can establish infection.

To summarize, longer acting drugs are more robust to the effects of imperfect adherence when drug resistance is expected to arise due to mutants present early on after the start of treatment. Including the effects of treatment non-adherence doesn't alter the relationship between the risk of resistance and the frequency of dosing that exists for perfect adherence. During a persistent infection, non-adherence can dramatically increase the risk of resistance unless we are in the regime where treatment fails due to the rebound of the WT infection.

\section{Multi-step mutations}

So far we have considered a model with only two viral strains (WT and the resistant mutant), where one effective mutation rate encapsulates the effects of all the genetic changes needed for the wild-type to acquire drug resistance. In reality, resistance is often attained via step-wise mutations [58,59] where the path to resistance is via intermediate strains. These mutational pathways can affect the role played by drug kinetics on the emergence of resistance. Long term exposure to sub-optimal drug concentrations can play a major role in facilitating the emergence of step-wise resistance when the intermediate strains are susceptible to the drug. In order to investigate whether mutational pathways can affect the relationship between drug dosing intervals and the risk of resistance observed so far, we used a standard extension of the viral dynamics model that includes intermediate viral strains [60,61]. The model consists of the WT strain, two intermediate strains that are one step mutation away from the WT and a resistant strain that is a step and two-step mutation away from the intermediate and WT strains respectively (Suppl. Methods). In the absence of treatment, the WT is the fittest strain (Figure 5A). We assume that the WT and the intermediate strains are suppressed by treatment (with the intermediate strains having the same fitness as each other) and the resistant strain is not (Figure 5A).

The establishment probability of one pre-existing resistant mutant is the same irrespective of the number of step-wise mutations needed from the WT to acquire resistance. This is because the long term fate (establishment probability) of the mutant is independent of how it was produced. As a result, mutational pathways don't play a role and the effect of dosing intervals on the risk of resistance due to a two-step pre-existing resistant mutant is the same as in Figure 2.

On the other hand, the establishment of a resistant infection due to rescue mutants depends upon the rate of production from the wild-type and intermediate strains and consequently depends upon the mutational pathway (Suppl. Methods). We analyzed the rescue probability (Figure 5B) of a partially resistant mutant as a function of the drug dosing intervals for the pharmacologically-inspired drug efficacy model for perfect and imperfect adherence. We find that the trends are the same as that for one-step mutants, with long-acting therapies corresponding to a lower risk of resistance.

We also calculated the risk of resistance during a persistent infection for the two-step mutant for both 
medRxiv preprint doi: https://doi.org/10.1101/2021.07.10.21260044; this version posted July 14, 2021. The copyright holder for this preprint (which was not certified by peer review) is the author/funder, who has granted medRxiv a license to display the preprint in perpetuity.

It is made available under a CC-BY 4.0 International license.

A

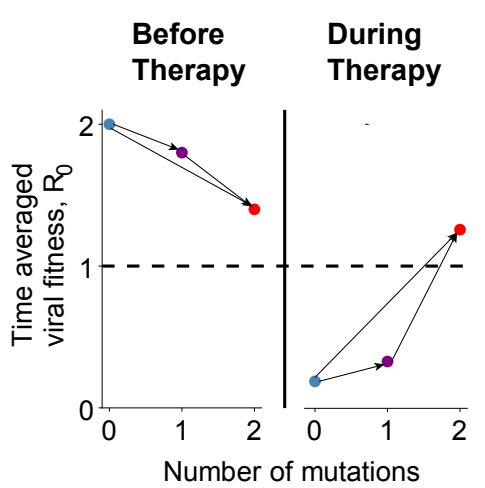

C

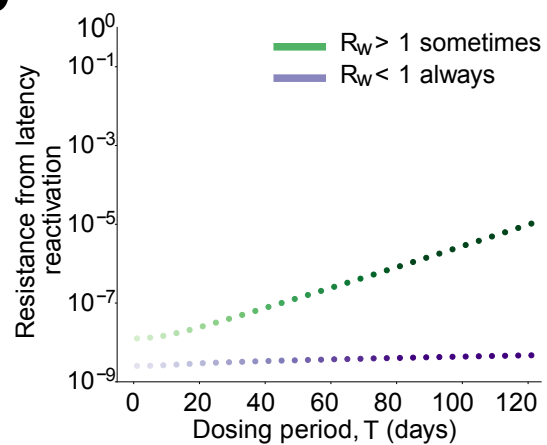

B

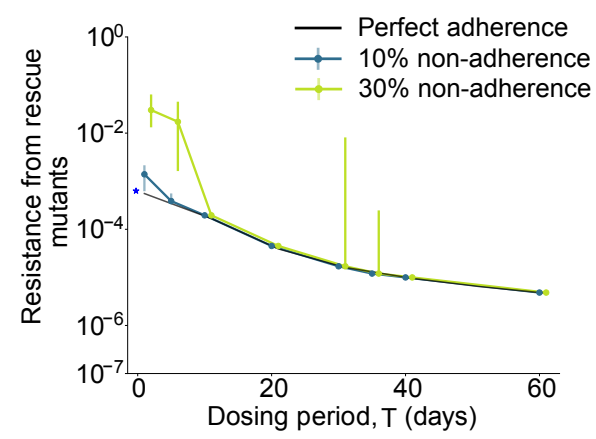

D

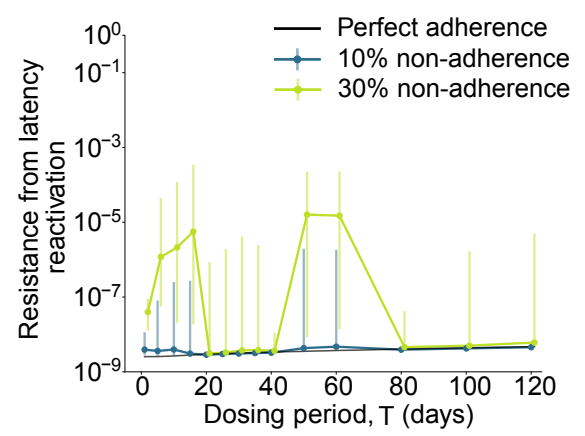

Figure 5: Effect of drug dosing intervals on the establishment of resistance for two-step mutants. Results are for a two-step partially resistant mutant $\left(\rho_{r}=2\right)$ and pharmacologically-inspired efficacy model. A) Time-averaged viral fitness of the WT (blue dot), intermediate strains (purple dot) and resistant strain (red dot), before and during treatment. B) Probability that at least one rescue mutant is produced as a function of the dosing interval for perfect (black line) and imperfect adherence. The blue starred point corresponds to the rescue probability for constant efficacy: $\epsilon_{\mathrm{avg}}=\langle\epsilon(t)\rangle(\mathrm{blue}$ star). C) Average probability of rescue per latency reactivation event as a function of the dosing interval for two strengths of the efficacy profile with perfect adherence. For the green curve, $R_{0}$ for the WT $>1$ for $\sim 23 \%$ of the cycle and $>1$ for $\sim 35 \%$ of the cycle for the intermediate mutants. D) Effect of imperfect adherence on the average probability of rescue per latency reactivation event. The black line corresponds to perfect adherence for the strong drug efficacy from C). Parameter values, $C_{\max }=20, C_{\max }=17$ (only for the green curve in B), $I C_{50}=10, M=10, \frac{t_{h}}{T}=1, \rho_{w}=1, \rho_{m}=1.1$. Results are medians of 100 iterations for rescue and 500 iterations for latency with the error bars corresponding to the interquartile range. 
perfect (Figure 5C) and imperfect adherence (Figure 5D). Even in this case, the trends are the same as that for one-step mutants.

Overall, we find that the mutational pathway considered here doesn't change the qualitative relationship between the drug dosing intervals and the risk of resistance that exists for the simplified case of a one-step mutant.

\section{Discussion}

New long-acting antimicrobial therapies are being developed to improve patient adherence and reduce treatment failure [11]. Although these drug formulations are likely to reduce missed doses, it is unclear whether this alone will lead to better treatment outcomes. Therapies with extended half-lives can lead to long-term exposure to intermediate drug levels, which could promote the evolution of drug resistance [34,35]. A systematical study of the relationship between drug dosing kinetics and the risk of resistance is therefore essential to the development and optimization of new long-acting therapies.

In this study we found that the interplay between time-varying drug concentrations in the body and competing pathogen strains results in a complicated effect of drug dosing intervals on the evolution of resistance (Table 1). Using models of infection dynamics within hosts [37,52,53], we show that when resistance mutations pre-exist before treatment and confer complete resistance, long-acting therapies increase the resistance risk if drug absorption is fast and reduce it if drug absorption is slow. These trends can be reversed if pre-existing mutants confer only partial resistance. On the other hand, when the main source of resistance is mutants produced de novo via residual wild-type infection after treatment begins ("rescue"), then irrespective of the degree of resistance long-acting drugs reduce the resistance risk if drug absorption is fast and increase it if drug absorption is slow. Our results demonstrate that resistance risk due to both pre-existing and rescue mutations is dominated by drug kinetics and infection dynamics in a short initial time window after treatment begins, which we call the "establishment time frame".

\begin{tabular}{|c|c|c|c|}
\hline & & Degree of 1 & istance \\
\hline Source of resistance & Drug kinetics model & Full & Partial \\
\hline Pre-existing & Slow drug absorption & $\downarrow$ & $\uparrow \downarrow$ \\
\hline & Fast drug absorption & $\uparrow$ & $\downarrow \uparrow$ \\
\hline De novo & Slow drug absorption & $\uparrow$ & $\uparrow$ \\
\hline & Fast drug absorption & $\downarrow$ & $\downarrow$ \\
\hline Latency reactivation & Suppressive minimum drug level & No change & No change \\
\hline & Non-suppressive minimum drug level & $\uparrow$ & $\uparrow$ \\
\hline
\end{tabular}

Table 1: Summary of effect of long-acting drugs on the risk of resistance during therapy. "个" means that the probability of drug resistance emerging during long-acting therapy is higher compared to daily dosing (with the same average, peak, and minimum levels), and " $\downarrow$ " means the opposite. Smaller sized arrows indicate minimal effect. For resistance arising from either mutations that are "pre-existing" at the time of therapy initiation or from mutations produced "de novo" by residual replication before wild-type infection is suppressed, the speed of drug absorption (relative to clearance) is the important pharmacokinetic factor determining resistance risk. Fast absorption means that the peak drug level occurs shortly after the dose is taken. The effect on pre-existing partially resistant mutants changes depending upon which of the two selection forces (competition with the WT for available target cells and the suppression of the resistant strain by the drug) dominates. For resistance arising from reactivation of persistent infection long after therapy initiation, the inhibitory effect of the minimum drug level is the most important pharmacokinetic factor determining resistance risk. Results come from simulations with a specific infection model and parameter values, and while our analyses suggest trends are relatively general, the magnitude of the effect will change with model details.

Our analysis essentially amounts to calculating the fixation probability of a mutant under time-varying selection pressures. This problem has been studied in the population genetics literature, but is complicated 
to analyze unless the timescales of environmental change and fixation are very different. When environmental changes occur much faster than the time from mutant appearance to fixation, the time-averaged fitness of the mutant determines the probability of fixation [62]. At the other extreme, when environmental changes occur slowly as compared to the time-scale of selection, the fixation probability is governed by the environmental conditions at the time of the mutant introduction [63]. We observe exactly the same effects for drug resistance, with the "establishment time frame" corresponding to the time-scale of fixation and the drug kinetics reflecting environmental change. The time-average drug efficacy can capture the fate of the resistant mutant when dosing is frequent, whereas the initial drug concentration determines the risk of resistance for very long acting drugs. As demonstrated by Cvijovic et al [38], in the intermediate regime where the two time-scales are comparable, the dynamics of the environmental fluctuations (drug kinetics in our case) strongly influence the fixation of the mutant and can't be explained by any effective selection pressure alone.

Drug resistance mutations may completely abrogate dose-dependent inhibition by the drug, or more commonly, confer a partial reduction in the drug efficacy. We found that the fate of pre-existing mutations under long-acting therapy depended strongly on the degree of resistance. Counter-intuitively, we found that sustained higher drug levels at the initiation of therapy did not necessarily correspond to lower resistance risks, since this could lead to more rapid removal of the wild-type strain, which competes for resources with the resistant strain. This result is in agreement with the more general observation from other modeling studies $[36,47,64]$ and experiments $[65,66]$ showing that aggressive treatment doesn't necessarily lead to reduced resistance risk. In contrast, we observed that the de novo generation and fixation of resistance mutations was effectively suppressed by long-acting therapies that were absorbed quickly to high levels .

Treatment for infections such as HIV and TB can be compromised by persistent sources of infection which aren't rapidly cleared by therapy. During such infections, early drug kinetics become less important. Resistant mutants can arise at any time from pre-existing mutant populations in the persistor pool, or get generated during breakthrough replication of WT cells from the persistent population when drug levels are low. We found that during persistent infection, longer-acting therapies increase the risk of resistance if the drug isn't fully suppressive during the lowest drug levels. Long-term exposure to low drug levels can allow WT populations that reactivate from latency to temporarily grow, significantly increasing the chance that resistant mutants are generated and expand. Individual physiological differences in drug absorption, distribution, metabolism, and excretion mean that the suppressive action of therapy can vary significantly across a population, and previous studies have stressed the need to individualize treatment regimens [40,67]. Our findings suggest that this need might be even more acute for longer-acting therapies.

Imperfect treatment adherence is a major challenge for long-term antimicrobial therapy and can facilitate the emergence of resistance. While the development of long-acting therapies was motivated by the need to improve adherence, their robustness to missed doses has not been evaluated. Mathematical models have helped provide valuable insights into understanding the complex and at times surprising effects of treatment non-adherence [39-45,68]. Extending this work to long-acting therapies, we find that the effects of imperfect adherence depend on whether there is a persistent source of infection. In its absence, long-acting drugs are more robust to the effects of imperfect adherence compared to more frequent dosing, since it is less likely doses will be missed in the critical early time frame during which wild-type infection is cleared before resistant strains can be produced or effectively compete for resources. However, if there is a persistent population from which infection can reactivate during later periods of non-adherence, then some intermediate dosing frequencies lead to dramatically higher resistance risks.

Our model assumes a single well-mixed pathogen population. In reality, pathogen subpopulations can reside in tissues and organs [69,70] where drug absorption and pathogen dynamics may be significantly altered [71-73]. Previous work has considered the consequences of spatial heterogeneity on treatment efficacy and the role it can play in facilitating the emergence of drug resistance [74-80]. In our analysis, the rate of drug absorption played an important role in determining the risk of resistance to long-acting therapies. Since drug absorption and clearance can differ between tissues, we suspect that the relationship between the frequency of dosing and the emergence of resistance could be more complex for compartmentalized infections.

Currently, injectable antiretroviral therapy combining cabotegravir and rilpivirine is the only long-acting antimicrobial therapy in late-stage of clinical trials $[12,14-16]$. Preliminary results from these trials indicate non-inferiority of the long-acting formulation for maintaining HIV suppression, compared to the current standard of care consisting of a daily oral dosing [81]. Our analysis provides some insight into what to 
expect in the long-term. In these trials, participants initiating therapy were given standard daily doses until viral suppression was achieved (less than 50 viral RNA copies per $\mathrm{mL}$ ) after which they were randomly assigned to a longer dosing schedule (4 or 8 weeks) or kept at daily dosing as a control. Our analysis suggests that by switching to longer-acting therapy only after viral suppression is achieved, the risk of resistance from pre-existing or rescue mutations during the critical establishment time frame may be negated. HIV infection will still persist indefinitely in the the latent reservoir and may reactivate during periods of non-adherence, but results suggest that long-acting therapy will not be associated with an increased risk of resistance in these trials if drug concentration levels are kept high enough in all individuals to ensure WT suppression even at the lowest points of the drug-cycle. According to the results of pharmacokinetic studies so far, this indeed seems to be the case $[12,14-16]$.

Long-acting drugs are also being considered for prophylactic use to improve adherence and increase effectiveness in preventing infection, for example for HIV and malaria [19,82-84]. For long-acting preventative therapy, there are concerns about the emergence of resistance due to long-term exposure of intermediate drug levels if prophylaxis is insufficient to prevent infection, or is initiated during an undiagnosed acute infection [85,86]. Penrose et al [86] report an instance of infection with wild-type (WT) HIV and subsequent selection of a resistant virus due to persistent exposure to long-acting pre-exposure prophylaxis (PrEP). In addition, Radzio-Basu et al [85] show in a macaque model that initiating long-acting PrEP during acute simian-human immunodeficiency virus (SHIV) infection can frequently select mutations conferring resistance to therapy and are maintained for several months. Although we have not considered prophylactic use directly, some of our results seem qualitatively applicable. The risk of resistance if prophylaxis is unknowingly started during acute infection may be similar to our findings for the risk due to de novo mutations after therapy initiation. The risk of resistance during breakthrough infections is likely to depend on drug-kinetics in a similar way to that of reactivating persistent infection, but the chance of breakthrough infection itself may require a more specific model of transmission.

To conclude, our results demonstrate that long-acting antimicrobial therapies can reduce resistance risk in certain settings, but can also increase risks despite improving adherence. The emergence of resistance tends to be more likely if long-acting drugs are slowly absorbed, not fully suppressive in all individuals at minimum concentrations, and if reactivation of persistent infection is a continual risk. Our findings underscore the importance of explicitly modeling drug kinetics, since time-averaged drug efficacy is a poor proxy for resistance risk. Future work should focus on developing application-specific models that use data-driven pharmacodynamic, pharmacokinetic, and evolutionary parameters. Such models can help guide future dose-optimization and implementation tasks for long-acting therapies for globally important infectious diseases.

\section{References}

[1] Arts E, Hazuda D. HIV-1 antiretroviral drug therapy. Cold Spring Harbor Perspectives in Medicine. 2012;2(4):7681-7686. Pmid:22474613.

[2] Rajbhandari R, Chung R. Treatment of Hepatitis B: A Concise Review. Clinical and Translational Gastroenterology. 2016;7(9):e190. Pmid:27628420.

[3] Webster D, Klenerman P, Dusheiko G. Hepatitis C. The Lancet. 2015;385(9973):1124-1135.

[4] Johnson V, Calvez V, Günter H, Paredes R, Pillay D, Shafer R, et al. 2011 update of the drug resistance mutations in HIV- 1. Topics in Antiviral Medicine. 2011;19:156-164.

[5] Maldarelli F, Kearney M, Palmer S, Stephens R, Mican J, Polis MA, et al. HIV Populations Are Large and Accumulate High Genetic Diversity in a Nonlinear Fashion. Journal of Virology. 2013 Sep;87(18):10313-10323. Available from: https://www.ncbi.nlm.nih.gov/pmc/articles/ PMC3754011/.

[6] Cuevas J, Geller R, Garijo R, López-Aldeguer J, Sanjuán R. Extremely High Mutation Rate of HIV-1 In Vivo. PLoS Biol. 2015;13(9):e1002251. 
[7] Goldberg D, Siliciano R, Jacobs Jr W. Outwitting Evolution: Fighting Drug-Resistant TB, Malaria, and HIV. Cell. 2012 Mar;148(6):1271-1283. Available from: http://www.sciencedirect.com/science/ article/pii/S0092867412002218.

[8] DiMatteo M, Giordani P, Lepper H, Croghan T. Patient adherence and medical treatment outcomes: a meta-analysis. Med Care. 2002;40(9):794-811.

[9] Paterson D, Swindells S, Mohr J, Brester M, Vergis E, Squier C, et al. Adherence to protease inhibitor therapy and outcomes in patients with HIV infection. Ann Intern Med. 2000;133(1):21-30.

[10] Allard NL, MacLachlan JH, Dev A, Dwyer J, Srivatsa G, Spelman T, et al. Adherence in chronic hepatitis B: associations between medication possession ratio and adverse viral outcomes. BMC Gastroenterology. 2020 May;20. Available from: https://www.ncbi.nlm.nih.gov/pmc/articles/PMC7203797/.

[11] Flexner C, Thomas DL, Swindells S. Creating demand for long-acting formulations for the treatment and prevention of HIV, tuberculosis, and viral hepatitis. Current Opinion in HIV and AIDS. 2019 Jan;14(1):13-20. Available from: https://journals.lww.com/01222929-201901000-00004.

[12] Margolis D, Gonzalez-Garcia J, Stellbrink H, Eron J, Yazdanpanah Y, Podzamczer D, et al. Long-acting intramuscular cabotegravir and rilpivirine in adults with hiv-1 infection (latte-2): 96-week results of a randomised, open-label, phase 2b, non-inferiority trial. The Lancet. 2017;390(10101):1499-1510.

[13] Gulick R, Flexner C. Long-Acting HIV Drugs for Treatment and Prevention. Annu Rev Med. 2019;70:137-150.

[14] Orkin C, Arasteh K, Górgolas Hernández-Mora M, Pokrovsky V, Overton ET, Girard PM, et al. Long-Acting Cabotegravir and Rilpivirine after Oral Induction for HIV-1 Infection. New England Journal of Medicine. 2020 Mar;382(12):1124-1135. Publisher: Massachusetts Medical Society _eprint: https://doi.org/10.1056/NEJMoa1909512. Available from: https://doi.org/10.1056/ NEJMoa1909512.

[15] Swindells S, Andrade-Villanueva JF, Richmond GJ, Rizzardini G, Baumgarten A, Masiá M, et al. Long-Acting Cabotegravir and Rilpivirine for Maintenance of HIV-1 Suppression. New England Journal of Medicine. 2020 Mar;382(12):1112-1123. Publisher: Massachusetts Medical Society _eprint: https://doi.org/10.1056/NEJMoa1904398. Available from: https://doi.org/10.1056/ NEJMoa1904398.

[16] Overton ET, Richmond G, Rizzardini G, Jaeger H, Orrell C, Nagimova F, et al. Long-acting cabotegravir and rilpivirine dosed every 2 months in adults with HIV-1 infection (ATLAS-2M), 48-week results: a randomised, multicentre, open-label, phase 3b, non-inferiority study. The Lancet. 2020 Dec;396(10267):1994-2005. Available from: https://www.sciencedirect.com/science/article/ $\mathrm{pii/S0140673620326660.}$

[17] University of Liverpool. $\$ 24 \mathrm{~m}$ boost for revolutionary long-acting medicine research. University of Liverpool News. 2020 Jan;Available from: https://news.liverpool.ac.uk/2020/01/31/ 24m-boost-for-revolutionary-long-acting-medicine-research/.

[18] Swindells S, Siccardi M, Barrett SE, Olsen DB, Grobler JA, Podany A, et al. Long-acting formulations for the treatment of latent tuberculous infection: opportunities and challenges. The international journal of tuberculosis and lung disease : the official journal of the International Union against Tuberculosis and Lung Disease. 2018 Feb;22(2):125-132. Available from: https://www.ncbi.nlm.nih.gov/pmc/ articles/PMC6103451/.

[19] Bakshi RP, Tatham LM, Savage AC, Tripathi AK, Mlambo G, Ippolito MM, et al. Long-acting injectable atovaquone nanomedicines for malaria prophylaxis. Nature Communications. 2018 Jan;9(1):315. Number: 1 Publisher: Nature Publishing Group. Available from: https://www.nature.com/articles/ s41467-017-02603-z. 
medRxiv preprint doi: https://doi.org/10.1101/2021.07.10.21260044; this version posted July 14, 2021. The copyright holder for this preprint

(which was not certified by peer review) is the author/funder, who has granted medRxiv a license to display the preprint in perpetuity.

It is made available under a CC-BY 4.0 International license .

[20] Verma M, Chu JN, Salama JAF, Faiz MT, Eweje F, Gwynne D, et al. Development of a long-acting direct-acting antiviral system for hepatitis $\mathrm{C}$ virus treatment in swine. Proceedings of the National Academy of Sciences. 2020 Jun;117(22):11987-11994. Publisher: National Academy of Sciences Section: Physical Sciences. Available from: https://www.pnas.org/content/117/22/11987.

[21] Kaushik A, Ammerman NC, Tyagi S, Saini V, Vervoort I, Lachau-Durand S, et al. Activity of a LongActing Injectable Bedaquiline Formulation in a Paucibacillary Mouse Model of Latent Tuberculosis Infection. Antimicrobial Agents and Chemotherapy. 2019 Apr;63(4). Publisher: American Society for Microbiology Journals Section: Experimental Therapeutics. Available from: https://aac.asm.org/ content/63/4/e00007-19.

[22] McCarthy MW, Keyloun KR, Gillard P, Choi JJ, Pickell N, Copp R, et al. Dalbavancin Reduces Hospital Stay and Improves Productivity for Patients with Acute Bacterial Skin and Skin Structure Infections: The ENHANCE Trial. Infectious Diseases and Therapy. 2020 Mar;9(1):53-67. Available from: https://doi.org/10.1007/s40121-019-00275-4.

[23] Vazquez D, Axel A, Shihadeh KC, Preslaski CR, Young HL, Wyles DL, et al. Use of a Standardized Dalbavancin Approach to Facilitate Earlier Hospital Discharge for Vulnerable Patients Receiving Prolonged Inpatient Antibiotic Therapy. Open Forum Infectious Diseases. 2020 Aug;7(ofaa293). Available from: https://doi.org/10.1093/ofid/ofaa293.

[24] Morrisette T, Miller MA, Montague BT, Barber GR, McQueen RB, Krsak M. Long-Acting Lipoglycopeptides: "Lineless Antibiotics" for Serious Infections in Persons Who Use Drugs. Open Forum Infectious Diseases. 2019 Jul;6(ofz274). Available from: https://doi.org/10.1093/ofid/ofz274.

[25] Salazar G, Zhang N, Fu TM, An Z. Antibody therapies for the prevention and treatment of viral infections. npj Vaccines. 2017 Jul;2(1):1-12. Number: 1 Publisher: Nature Publishing Group. Available from: https://www.nature.com/articles/s41541-017-0019-3.

[26] Bangsberg DR, Kroetz DL, Deeks SG. Adherence-resistance relationships to combination HIV antiretroviral therapy. Current HIV/AIDS Reports. 2007 May;4(2):65-72. Available from: http: //www.ncbi.nlm.nih.gov/pubmed/17547827.

[27] Maggiolo F, Airoldi M, Kleinloog HD, Callegaro A, Ravasio V, Arici C, et al. Effect of adherence to HAART on virologic outcome and on the selection of resistance-conferring mutations in NNRTI- or PI-treated patients. HIV Clinical Trials. 2007 Oct;8(5):282-292. Available from: http://www.ncbi. nlm.nih.gov/pubmed/17956829.

[28] Von Wyl V, Klimkait T, Yerly S, Nicca D, Furrer H, Cavassini M, et al. Adherence as a Predictor of the Development of Class-Specific Resistance Mutations: The Swiss HIV Cohort Study. PLoS ONE. 2013 Oct;8(10):e77691. Available from: http://dx.doi.org/10.1371/journal.pone.0077691.

[29] Mitchison DA. How drug resistance emerges as a result of poor compliance during short course chemotherapy for tuberculosis [Counterpoint]. The International Journal of Tuberculosis and Lung Disease. 1998;2(1):10-15. Available from: http://www. ingentaconnect.com/content/iuatld/ijtld/ 1998/00000002/00000001/art00005.

[30] Law S, Piatek AS, Vincent C, Oxlade O, Menzies D. Emergence of drug resistance in patients with tuberculosis cared for by the Indian health-care system: a dynamic modelling study. The Lancet Public Health. 2017 Jan;2(1):e47-e55. Publisher: Elsevier. Available from: https://www.thelancet.com/ journals/lanpub/article/PIIS2468-2667(16)30035-4/abstract.

[31] Rockwood N, Abdullahi LH, Wilkinson RJ, Meintjes G. Risk Factors for Acquired Rifamycin and Isoniazid Resistance: A Systematic Review and Meta-Analysis. PLOS ONE. 2015 Sep;10(9):e0139017. Publisher: Public Library of Science. Available from: https://journals.plos.org/plosone/article? id $=10.1371 /$ journal . pone .0139017. 
[32] Wang L, Chen P, Zheng C. Poor adherence is a contributor to viral breakthrough in patients with chronic hepatitis B. Infection and Drug Resistance. 2018 Nov;11:2179-2185. Available from: https: //www.ncbi.nlm.nih.gov/pmc/articles/PMC6233945/.

[33] Mohd Shariff N, Shah SA, Kamaludin F. Previous treatment, sputum-smear nonconversion, and suburban living: The risk factors of multidrug-resistant tuberculosis among Malaysians. International Journal of Mycobacteriology. 2016 Mar;5(1):51-58.

[34] Andersson DI, Hughes D. Microbiological effects of sublethal levels of antibiotics. Nature Reviews Microbiology. 2014 Jul;12(7):465-478. Number: 7 Publisher: Nature Publishing Group. Available from: http://www. nature. com/articles/nrmicro3270.

[35] Hastings Ian M , Watkins William M, White Nicholas J . The evolution of drug-resistant malaria: the role of drug elimination half-life. Philosophical Transactions of the Royal Society of London Series B: Biological Sciences. 2002 Apr;357(1420):505-519. Available from: https://royalsocietypublishing. org/doi/abs/10.1098/rstb.2001.1036.

[36] Bonhoeffer S, May RM, Shaw GM, Nowak MA. Virus dynamics and drug therapy. Proceedings of the National Academy of Sciences. 1997;94(13):6971-6976. Available from: https://www.pnas.org/ content/94/13/6971.

[37] Alexander H, Bonhoeffer S. Pre-existence and emergence of drug resistance in a generalized model of intra-host viral dynamics. Epidemics. 2012;4(4):187-202.

[38] Cvijović I, Good BH, Jerison ER, Desai MM. Fate of a mutation in a fluctuating environment. Proceedings of the National Academy of Sciences. 2015 Sep;112(36):E5021-E5028. Available from: http://www. pnas.org/content/112/36/E5021.

[39] Wahl LM, Nowak MA. Adherence and drug resistance: predictions for therapy outcome. Proceedings of the Royal Society B: Biological Sciences. 2000 Apr;267(1445):835 -843. Available from: http://rspb. royalsocietypublishing.org/content/267/1445/835. abstract.

[40] Wu H, Huang Y, Acosta EP, Rosenkranz SL, Kuritzkes DR, Eron JJ, et al. Modeling long-term HIV dynamics and antiretroviral response: effects of drug potency, pharmacokinetics, adherence, and drug resistance. Journal of Acquired Immune Deficiency Syndromes (1999). 2005 Jul;39(3):272-283. Available from: http://www.ncbi.nlm.nih.gov/pubmed/15980686.

[41] Krakovska O, Wahl LM. Optimal drug treatment regimens for HIV depend on adherence. Journal of Theoretical Biology. 2007 Jun;246(3):499-509. Available from: http://www.sciencedirect.com/ science/article/pii/S0022519306006047.

[42] Huang Y, Wu H, Holden-Wiltse J, Acosta EP. A dynamic Bayesian nonlinear mixed-effects model of HIV response incorporating medication adherence, drug resistance and covariates. The annals of applied statistics. 2011;5(1):551. Available from: http://www.ncbi.nlm.nih.gov/pmc/articles/ PMC3498098/.

[43] Cadosch D, Bonhoeffer S, Kouyos R. Assessing the impact of adherence to anti-retroviral therapy on treatment failure and resistance evolution in HIV. Journal of The Royal Society Interface. 2012 Sep;9(74):2309-2320. Available from: http://rsif.royalsocietypublishing.org/content/9/74/ 2309.

[44] Rosenbloom DIS, Hill AL, Rabi SA, Siliciano RF, Nowak MA. Antiretroviral dynamics determines HIV evolution and predicts therapy outcome. Nature Medicine. 2012;18(9):1378-1385. Available from: http://www. nature.com.ezp-prod1.hul. harvard.edu/nm/journal/v18/n9/full/nm.2892.html.

[45] Bershteyn A, Eckhoff PA. A model of HIV drug resistance driven by heterogeneities in host immunity and adherence patterns. BMC Systems Biology. 2013;7:11. Available from: http://dx.doi.org/10. 1186/1752-0509-7-11. 
medRxiv preprint doi: https://doi.org/10.1101/2021.07.10.21260044; this version posted July 14, 2021. The copyright holder for this preprint

(which was not certified by peer review) is the author/funder, who has granted medRxiv a license to display the preprint in perpetuity.

It is made available under a CC-BY 4.0 International license .

[46] Conway JM, Perelson AS. A hepatitis C virus infection model with time-varying drug effectiveness: solution and analysis. PLoS computational biology. 2014 Aug;10(8):e1003769.

[47] Colijn C, Cohen T. How competition governs whether moderate or aggressive treatment minimizes antibiotic resistance. eLife. 2015;4:e10559. Available from: https://elifesciences.org/content/4/ e10559v3.

[48] Schiffer JT, Swan DA, Magaret A, Corey L, Wald A, Ossig J, et al. Mathematical modeling of herpes simplex virus-2 suppression with pritelivir predicts trial outcomes. Science Translational Medicine. 2016 Feb;8(324):324ra15.

[49] Best K, Guedj J, Madelain V, Lamballerie Xd, Lim SY, Osuna CE, et al. Zika plasma viral dynamics in nonhuman primates provides insights into early infection and antiviral strategies. Proceedings of the National Academy of Sciences. 2017 Aug;114(33):8847-8852. Available from: http://www.pnas.org/ content/114/33/8847.

[50] Madelain V, Baize S, Jacquot F, Reynard S, Fizet A, Barron S, et al. Ebola viral dynamics in nonhuman primates provides insights into virus immuno-pathogenesis and antiviral strategies. Nature Communications. 2018 Oct;9(1):4013. Number: 1 Publisher: Nature Publishing Group. Available from: https://www. nature.com/articles/s41467-018-06215-z.

[51] Lingas G, Rosenke K, Safronetz D, Guedj J. Lassa viral dynamics in non-human primates treated with favipiravir or ribavirin. PLOS Computational Biology. 2021 Jan;17(1):e1008535. Publisher: Public Library of Science. Available from: https://journals.plos.org/ploscompbiol/article?id=10.1371/ journal.pcbi.1008535.

[52] Nowak M, May R. Virus dynamics: Mathematical principles of immunology and virology. Oxford University Press; 2000.

[53] Rong L, Feng Z, Perelson A. Emergence of HIV-1 Drug Resistance During Antiretroviral Treatment. Bulletin of mathematical biology. 2007 09;69:2027-60.

[54] Bell G, Gonzalez A. Evolutionary rescue can prevent extinction following environmental change. Ecology Letters. 2009;12(9):942-948. Available from: https://onlinelibrary.wiley.com/doi/abs/10.1111/ j.1461-0248.2009.01350.x.

[55] Orr HA, Unckless RL. Population Extinction and the Genetics of Adaptation. The American Naturalist. 2008;172(2):160-169.

[56] Hill AL. Mathematical Models of HIV Latency. In: Silvestri G, Lichterfeld M, editors. HIV Latency. Current Topics in Microbiology and Immunology. Springer, Berlin, Heidelberg; 2017. p. 1-26. Available from: https://link.springer.com/chapter/10.1007/82_2017_77.

[57] Raimondo G, Pollicino T, Cacciola I, Squadrito G. Occult hepatitis B virus infection. Journal of Hepatology. 2007 Jan;46(1):160-170. Publisher: Elsevier. Available from: https://www . journal-of-hepatology.eu/article/S0168-8278(06)00577-0/abstract.

[58] Shockley TE, Hotchkiss RD. Stepwise introduction of transformable penicillin resistance in pneumococcus. Genetics. 1970 Mar;64(3-4):397-408. Available from: https://academic.oup.com/genetics/ article/64/3-4/397-408/5989862.

[59] Bryan LE. Antimicrobial Drug Resistance. Academic Press; 1984. Available from: https://books . google.com/books?id=5RVrAAAAMAAJ.

[60] Boerlijst MC, Bonhoeffer S, Nowak MA. Viral quasi-species and recombination. Proceedings of the Royal Society of London Series B: Biological Sciences. 1996 Nov;263(1376):1577-1584. Publisher: Royal Society. Available from: https://royalsocietypublishing.org/doi/10.1098/rspb.1996.0231. 
medRxiv preprint doi: https://doi.org/10.1101/2021.07.10.21260044; this version posted July 14, 2021. The copyright holder for this preprint

(which was not certified by peer review) is the author/funder, who has granted medRxiv a license to display the preprint in perpetuity.

It is made available under a CC-BY 4.0 International license .

[61] Ribeiro RM, Bonhoeffer S, Nowak MA. The frequency of resistant mutant virus before antiviral therapy. AIDS. 1998 Mar;12(5):461-465. Available from: https://journals.lww.com/aidsonline/Fulltext/ 1998/05000/The_frequency_of_resistant_mutant_virus_before.6.aspx.

[62] Mustonen V, Lässig M. From fitness landscapes to seascapes: non-equilibrium dynamics of selection and adaptation. Trends in Genetics. 2009 Mar;25(3):111-119. Available from: https://www . sciencedirect.com/science/article/pii/S0168952509000250.

[63] Mustonen V, Lässig M. Molecular Evolution under Fitness Fluctuations. Phys Rev Lett. 2008 Mar;100:108101. Available from: https://link.aps.org/doi/10.1103/PhysRevLett.100.108101.

[64] Day T, Read AF. Does High-Dose Antimicrobial Chemotherapy Prevent the Evolution of Resistance? PLOS Computational Biology. 2016 Jan;12(1):e1004689. Publisher: Public Library of Science. Available from: https://journals.plos.org/ploscompbiol/article?id=10.1371/journal.pcbi.1004689.

[65] Huijben S, Bell AS, Sim DG, Tomasello D, Mideo N, Day T, et al. Aggressive Chemotherapy and the Selection of Drug Resistant Pathogens. PLoS Pathogens. 2013 Sep;9(9). Available from: https: //www.ncbi.nlm.nih.gov/pmc/articles/PMC3771897/.

[66] Acosta MM, Bram JT, Sim D, Read AF. Effect of drug dose and timing of treatment on the emergence of drug resistance in vivo in a malaria model. Evolution, Medicine, and Public Health. 2020 06;2020(1):196210. Available from: https://doi.org/10.1093/emph/eoaa016.

[67] Andrew Clayton T, Lindon JC, Cloarec O, Antti H, Charuel C, Hanton G, et al. Pharmaco-metabonomic phenotyping and personalized drug treatment. Nature. 2006 Apr;440(7087):1073-1077. Number: 7087 Publisher: Nature Publishing Group. Available from: https://www.nature.com/articles/ nature 04648.

[68] Lipsitch M, Levin BR. Population dynamics of tuberculosis treatment: mathematical models of the roles of non-compliance and bacterial heterogeneity in the evolution of drug resistance. The International Journal of Tuberculosis and Lung Disease: The Official Journal of the International Union Against Tuberculosis and Lung Disease. 1998 Mar;2(3):187-199.

[69] Lorenzo-Redondo R, Fryer HR, Bedford T, Kim EY, Archer J, Pond SLK, et al. Persistent HIV-1 replication maintains the tissue reservoir during therapy. Nature. 2016 Feb;530(7588):51-56. Available from: https://www.ncbi.nlm.nih.gov/pmc/articles/PMC4865637/.

[70] Ganchua SKC, White AG, Klein EC, Flynn JL. Lymph nodes-The neglected battlefield in tuberculosis. PLoS pathogens. 2020 Aug;16(8):e1008632.

[71] Solas C, Lafeuillade A, Halfon P, Chadapaud S, Hittinger G, Lacarelle B. Discrepancies between protease inhibitor concentrations and viral load in reservoirs and sanctuary sites in human immunodeficiency virus-infected patients. Antimicrobial Agents and Chemotherapy. 2003 Jan;47(1):238-243.

[72] Else LJ, Taylor S, Back DJ, Khoo SH. Pharmacokinetics of antiretroviral drugs in anatomical sanctuary sites: the male and female genital tract. Antiviral Therapy. 2011;16(8):1149-1167.

[73] Nix DE, Goodwin SD, Peloquin CA, Rotella DL, Schentag JJ. Antibiotic tissue penetration and its relevance: impact of tissue penetration on infection response. Antimicrobial Agents and Chemotherapy. 1991 Oct;35(10):1953-1959. Available from: https://www.ncbi.nlm.nih.gov/pmc/articles/PMC245307/.

[74] Elliott AM, Berning SE, Iseman MD, Peloquin CA. Failure of drug penetration and acquisition of drug resistance in chronic tuberculous empyema. Tubercle and Lung Disease: The Official Journal of the International Union Against Tuberculosis and Lung Disease. 1995 Oct;76(5):463-467.

[75] Kepler TB, Perelson AS. Drug concentration heterogeneity facilitates the evolution of drug resistance. Proceedings of the National Academy of Sciences of the United States of America. 1998 Sep;95(20):11514-11519. Available from: https://www.ncbi.nlm.nih.gov/pmc/articles/PMC21672/. 
[76] Burkala EJ, He J, West JT, Wood C, Petito CK. Compartmentalization of HIV-1 in the central nervous system: role of the choroid plexus. AIDS (London, England). 2005 Apr;19(7):675-684.

[77] Hermsen R, Deris JB, Hwa T. On the rapidity of antibiotic resistance evolution facilitated by a concentration gradient. Proceedings of the National Academy of Sciences. 2012 Jul;109(27):1077510780. Publisher: National Academy of Sciences Section: Physical Sciences. Available from: https: //www.pnas.org/content/109/27/10775.

[78] Moreno-Gamez S, Hill AL, Rosenbloom DIS, Petrov DA, Nowak MA, Pennings PS. Imperfect drug penetration leads to spatial monotherapy and rapid evolution of multidrug resistance. Proceedings of the National Academy of Sciences of the United States of America. 2015 Jun;112(22):E2874-E2883. Available from: https://www.ncbi.nlm.nih.gov/pmc/articles/PMC4460514/.

[79] Gralka M, Fusco D, Martis S, Hallatschek O. Convection shapes the trade-off between antibiotic efficacy and the selection for resistance in spatial gradients. Physical biology. 2017 Jul;14(4):045011. Available from: https://www.ncbi.nlm.nih.gov/pmc/articles/PMC5728155/.

[80] Fuentes-Hernández A, Hernández-Koutoucheva A, Muñoz AF, Domínguez Palestino R, Peña-Miller R. Diffusion-driven enhancement of the antibiotic resistance selection window. Journal of the Royal Society Interface. 2019 Sep;16(158). Available from: https://www.ncbi.nlm.nih.gov/pmc/articles/ PMC6769300/.

[81] Phanuphak N, Gulick RM. HIV treatment and prevention 2019: current standards of care. Current opinion in HIV and AIDS. 2020 Jan;15(1):4-12.

[82] McPherson TD, Sobieszczyk ME, Markowitz M. Cabotegravir in the treatment and prevention of Human Immunodeficiency Virus-1. Expert Opinion on Investigational Drugs. 2018 Apr;27(4):413-420. Publisher: Taylor \& Francis _eprint: https://doi.org/10.1080/13543784.2018.1460357. Available from: https://doi.org/10.1080/13543784.2018.1460357.

[83] Markowitz M, Frank I, Grant RM, Mayer KH, Elion R, Goldstein D, et al. Safety and tolerability of long-acting cabotegravir injections in HIV-uninfected men (ECLAIR): a multicentre, double-blind, randomised, placebo-controlled, phase 2a trial. The lancet HIV. 2017 Aug;4(8):e331-e340.

[84] Landovitz RJ, Li S, Grinsztejn B, Dawood H, Liu AY, Magnus M, et al. Safety, tolerability, and pharmacokinetics of long-acting injectable cabotegravir in low-risk HIV-uninfected individuals: HPTN 077, a phase 2a randomized controlled trial. PLoS medicine. 2018 Nov;15(11):e1002690.

[85] Radzio-Basu J, Council O, Cong Me, Ruone S, Newton A, Wei X, et al. Drug resistance emergence in macaques administered cabotegravir long-acting for pre-exposure prophylaxis during acute SHIV infection. Nature Communications. 2019 May;10(1):2005. Number: 1 Publisher: Nature Publishing Group. Available from: https://www.nature.com/articles/s41467-019-10047-w.

[86] Penrose KJ, Parikh UM, Hamanishi KA, Else L, Back D, Boffito M, et al. Selection of RilpivirineResistant HIV-1 in a Seroconverter From the SSAT 040 Trial Who Received the 300-mg Dose of Long-Acting Rilpivirine (TMC278LA). The Journal of Infectious Diseases. 2016 Mar;213(6):1013-1017. Available from: https://doi.org/10.1093/infdis/jiv528.

[87] Sanjuán R, Nebot M, Chirico N, Mansky L, Belshaw R. Viral mutation rates. Journal of Virology. 2010;84:9733-9748.

[88] Loverdo C, Park M, Schreiber S, Lloyd-Smith J. Influence of viral replication mechanisms on within-host evolutionary dynamics. Evolution. 2012;66:3462-3471.

[89] Lloyd A. The dependence of viral parameter estimates on the assumed viral life cycle: limitations of studies of viral load data. Proceedings of the Royal Society B: Biological Sciences. 2001;268:847-854.

[90] Parzen E. Stochastic Processes. SIAM; 1999. 
medRxiv preprint doi: https://doi.org/10.1101/2021.07.10.21260044; this version posted July 14, 2021. The copyright holder for this preprint (which was not certified by peer review) is the author/funder, who has granted medRxiv a license to display the preprint in perpetuity.

It is made available under a CC-BY 4.0 International license.

[91] Wolfram Research, Inc . Mathematica, Version 12.0; 2020. Available from: https://www.wolfram. com/mathematica.

[92] Gillespie D. Approximate Accelerated Stochastic Simulation of Chemically Reacting Systems. Journal of Chemical Physics. 2001 07;115:1716-1733.

\section{Acknowledgements}

This work was supported by the Bill \& Melinda Gates Foundation (OPP1148627). The computations in this paper were run on the FASRC Cannon cluster supported by the FAS Division of Science Research Computing Group at Harvard University. We thank Giovanni Traverso, Ameya Kirtane, Morgan Craig, Martin Nowak, Katharine Best, Madison Ski Krieger, Michael Desai, and John Wakeley for helpful feedback on this project. 


\title{
Supplementary information : \\ The risk of drug resistance during long-acting antimicrobial therapy
}

\author{
Anjalika Nande ${ }^{1,2}$ and Alison L. Hill*1,2 \\ ${ }^{1}$ Program for Evolutionary Dynamics, Harvard University, Cambridge, MA 02138 \\ ${ }^{2}$ Institute for Computational Medicine, Johns Hopkins University, Baltimore, MD 21218
}

\section{Supplementary methods}

\section{Infection dynamics model}

\section{Before treatment}

We start from a standard ordinary differential equation model of intra-host viral dynamics [37,52] consisting of uninfected target cells $x$, cells infected by the drug-sensitive wild-type (WT) strain $y_{w}$, cells infected by the drug-resistant strain $y_{r}$, free $\mathrm{WT}$ virions $v_{w}$ and free resistant virions $v_{r}$,

$$
\begin{aligned}
\dot{x} & =\lambda-d x-\beta_{w}^{0} x v_{w}-\beta_{r}^{0} x v_{r} \\
\dot{y}_{w} & =(1-u) \beta_{w}^{0} x v_{w}-a_{w} y_{w} \\
\dot{y}_{r} & =u \beta_{w}^{0} x v_{w}+\beta_{r}^{0} x v_{r}-a_{r} y_{r} \\
\dot{v}_{w} & =k_{w} y_{w}-c_{w} v_{w} \\
\dot{v}_{r} & =k_{r} y_{r}-c_{r} v_{r}
\end{aligned}
$$

where $\lambda$ is the rate of target cell production (assumed to be constant for simplicity); $d$ is the natural death rate of target cells; $\beta_{w}^{0}$ and $\beta_{r}^{0}$ are the virus specific infectivities with the superscript indicating the value of the parameters in the absence of treatment; $a_{w}$ and $a_{r}$ are the death rates of the infected cells; $k_{w}$ and $k_{r}$ are the virion production rates; $c_{w}$ and $c_{r}$ are the free virion clearance rates; and $u$ is the mutation rate.

Mutation is modeled according to the 'stamping machine' mode of replication $[87,88]$ and back mutation is ignored. The results discussed in this paper don't depend upon when mutation occurs in a viral life-cycle and so we assume it occurs at the infection step without any loss of generality. Since we are modeling a system with only two strains, $u$ represents the effective mutation rate. It encapsulates the effect of all genetic changes that lead to resistance. This simplification ignores the effect of multi-step mutational pathways. We briefly discuss the effects of higher point mutants in later sections. However, this simple two-strain model is known to be good approximation when the fitness of the intermediate strains is low [37].

The fitness of the strains is summarized by their basic reproduction ratio, $R_{0} . R_{0}$ is defined as the average number of new infected cells produced in a single replication cycle by an infected cell introduced into a population of uninfected cells [52]. The WT reproduction ratio in the absence of drugs is given by,

$$
R_{0}^{w}=\frac{\lambda \beta_{w}^{0} k_{w}}{d a_{w} c_{w}}
$$

\footnotetext{
*Corresponding author: alhill@jhu.edu
} 
The resistant strain has some associated cost of resistance, $s$, that is incorporated such that $R_{0}^{r}=$ $(1-s) R_{0}^{w}$. We simplify the above model further by using the common assumption that the dynamics of the free virions are much faster than those of the infected cells [89]. This leads to quasi-equilibrium relationships $v_{w} \approx \frac{k_{w} y_{w}}{c_{w}}$ and $v_{r} \approx \frac{k_{r} y_{r}}{c_{r}}$ and the system Equation S1 becomes,

$$
\begin{aligned}
\dot{x} & \approx \lambda-d x-b_{w}^{0} x y_{w}-b_{r}^{0} x y_{r} \\
\dot{y}_{w} & \approx(1-u) b_{w}^{0} x y_{w}-a_{w} y_{w} \\
\dot{y}_{r} & \approx u b_{w}^{0} x y_{w}+b_{r}^{0} x y_{r}-a_{r} y_{r}
\end{aligned}
$$

where, $b_{i}^{0}=\frac{\beta_{i}^{0} k_{i}}{c_{i}}$.

At the infection equilibrium,

$$
\begin{aligned}
x^{\star} & =\frac{a_{w}}{b_{w}^{0}}=\frac{x_{0}}{R_{0}^{w}} \\
y_{w}^{\star} & =\frac{\lambda}{a_{w}}-\frac{d}{b_{w}^{0}} \\
y_{r}^{\star} & =\frac{a_{w} u}{a_{r} s} y_{w}^{\star}
\end{aligned}
$$

where $x_{0}=\frac{\lambda}{d}$ is the equilibrium level of target cells in the absence of infection. We assume for simplicity that the cost of resistance acts on viral infectivity, $b_{r}^{0}=(1-s) b_{w}^{0}$ and all other infection parameters for the two strains are the same.

\section{Incorporating treatment}

We model treatment by assuming that the drug blocks the infection of target cells. The infectivity parameter, $b$, becomes a function of the drug efficacy, $\epsilon$

$$
\begin{aligned}
\dot{x} & =\lambda-d x-b_{w}(t) x y_{w}-b_{r}(t) x y_{r} \\
\dot{y}_{w} & =(1-u) b_{w}(t) x y_{w}-a y_{w} \\
\dot{y}_{r} & =u b_{w}(t) x y_{w}+b_{r}(t) x y_{r}-a y_{r}
\end{aligned}
$$

where $b_{w}(t)=b_{w}^{0}\left(1-\epsilon_{w}(t)\right)$ and $b_{r}(t)=b_{r}^{0}\left(1-\epsilon_{r}(t)\right)$. We are interested in the case when the WT is suppressed in the presence of treatment, $R^{w}<1$ whereas the resistant strain is not $R^{r}>1$.

Figure S1 gives an example of what the dynamics look like when the treatment is initiated 100 days after the onset of infection. Prior to treatment, the system is at an equilibrium set by the WT. The resistant population is maintained at a low level via a mutation-selection balance. Once treatment starts, the WT is suppressed and the target cells start recovering. The resistant population decreases initially after treatment starts. This is on account of a lack of target cells to infect and a reduced influx from the suppressed WT population via mutation. The resistant population starts growing and establishes infection once the target cell availability is higher.

A deterministic approach guarantees the establishment of a resistant infection once the WT is suppressed during treatment, so long as $R^{r}>1$. However, mutations are inherently stochastic and the presence of just a few mutants prior to the start of treatment isn't enough to guarantee establishment as they can undergo stochastic extinction. In order to calculate the establishment probability of a resistant infection emerging during treatment, we use a stochastic version of the model. It is a continuous time Markov Chain with the transition rates given by the rates in the differential equations [37].

\section{Modeling latency}

To model latency as a source of resistance, we consider the scenario when the WT strain is completely suppressed by treatment and target cells have recovered to their pre-infection equilibrium $\left(x(0)=\frac{\lambda}{d}\right)$. WT 
infected cells are introduced into the system via reactivation of latent infected cells and continue to be suppressed by treatment. We assume that prior to treatment the mutant was maintained at a mutationselection balance $[52,61]$ which is reflected in the composition of the latent pool, a fraction $\left(\frac{u}{s}\right)$ of the latent pool consists of cells infected by the resistant strain. This leads to the following system of equations for the target cells $(x)$, WT $\left(y_{w}\right)$ and resistant mutant $\left(y_{r}\right)$ infected cells,

$$
\begin{aligned}
\dot{x} & =\lambda-d x-b_{w}(t) x y_{w}-b_{r}(t) x y_{r} \\
\dot{y}_{w} & =\gamma\left(1-\frac{u}{s}\right)+(1-u) b_{w}(t) x y_{w}-a y_{w} \\
\dot{y}_{r} & =\frac{\gamma u}{s}+u b_{w}(t) x y_{w}+b_{r}(t) x y_{r}-a y_{r}
\end{aligned}
$$

where $\gamma$ is the rate of latency reactivation and all other parameters are the same as in Equation S5.

\section{Infection parameter values}

\begin{tabular}{llll}
\hline Parameter & Description & Units & Value \\
\hline$\lambda$ & Rate of target cell production & cells/day & $2 \times 10^{6}$ \\
\hline$d$ & Death rate of target cells & day $^{-1}$ & 0.1 \\
\hline$k$ & Virion production rate & day $^{-1}$ & 1000 \\
\hline$a$ & Death rate of infected cells & day $^{-1}$ & 0.5 \\
\hline$c$ & Free virion clearance rate & day $^{-1}$ & 10 \\
\hline$\beta$ & Viral infectivity & day & \\
\hline & & & $5 \times 10^{-10}$ \\
\hline & Mutation rate & & \\
\hline & & & \\
\hline
\end{tabular}

Table S1: Infection parameter values used throughout this work unless stated otherwise

These parameters give a resulting $R_{0}=2$ for the wild-type and $R_{0}=1.4$ for the resistant mutant. We chose these parameter values to be consistent with Alexander and Bonhoeffer [37]. They provide their original sources and justify in detail why they are reasonable for the diseases of interest (for example HIV, HBV and $\mathrm{HCV}$ ). We stress that these values are only meant to qualitatively capture the nature of the infection dynamics. Pathogen specific values should be used in practice while evaluating the risks associated with new therapies. 


\section{Establishment probability}

We calculate the probability that a resistant mutant existing at a certain time after treatment initiation avoids extinction by modeling the process as a time inhomogeneous "Birth-Death" (B-D) process [90]. From Equation S5c births and deaths occur at the per capita rate,

$$
\begin{aligned}
B(t) & =b_{r}(t) x(t) \\
D & =a
\end{aligned}
$$

At the beginning of treatment, target and WT infected cells are present in very large numbers in contrast to the resistant mutant. So, we approximate that the target and WT infected cells follow deterministic dynamics given by the following simplified system of equations which can be solved numerically (we use Mathematica 12.0 [91]),

$$
\begin{aligned}
\dot{x} & =\lambda-d x-b_{w}(t) x y_{w} \\
\dot{y}_{w} & =b_{w}(t) x y_{w}-a y_{w}
\end{aligned}
$$

There exists an analytical solution for the extinction probability of such a B-D process [90],

$$
\operatorname{Pr}\left(y_{r}(t+T)=0 \mid y_{r}(t)=1\right)=\frac{\int_{t}^{t+T} D(\tau) e^{R(\tau)} \mathrm{d} \tau}{1+\int_{t}^{t+T} D(\tau) e^{R(\tau)} \mathrm{d} \tau}
$$

where $R(\tau)=\int_{t}^{\tau}\left(D\left(t^{\prime}\right)-B\left(t^{\prime}\right) \mathrm{d} t^{\prime}\right.$. The probability that the lineage of a strain existing at time $t$ eventually goes extinct is given by the limit,

$$
p_{\text {ext }}(t)=\lim _{T \rightarrow \infty} \operatorname{Pr}\left(y_{r}(t+T)=0 \mid y_{r}(t)=1\right)
$$

the establishment probability is,

$$
p_{\text {est }}(t)=1-p_{\text {ext }}(t)
$$

This "Birth-Death" approximation is used instead of stochastically simulating the whole model for each dosing interval as it captures the effects of stochasticity well and has the added advantage of calculating the risks significantly faster, within minutes as opposed to days. The approximation works well due to the deterministic nature of the target and WT infected cell dynamics coupled with the small population size of the resistant mutant at the onset of treatment. It does however have a limitation as it doesn't include the depletion of the target cells by the resistant strain (Equation S8). As a result, the establishment probability isn't well approximated when there is rapid growth of the resistant strain early on after treatment is initiated. But, this only affects the numerical results and doesn't change the qualitative trends associated with the risk of resistance and drug dosing intervals and the method can still be used to assess the relative risks associated with longer-acting therapies.

\section{Different sources of resistance}

Drug resistance can arise via different sources: mutants existing prior to treatment (pre-existing), those that are generated de novo during treatment via residual wild-type infection (rescue) and those that are a result of latency reactivation.

\section{Pre-existence}

The establishment probability for a single mutant present at the start of treatment is given by Equation S11 with $t=0$.

It is also possible to derive the total probability of survival due to pre-existence by taking into account the full distribution of the mutant population size prior to treatment. This can be approximated by a steady 
state birth-death-immigration (BDI) process [37]. The target and WT infected cell populations are large enough that fluctuations in their size aren't important and we can assume they are at their deterministic equilibrium level. This leads to a BDI process with constant rates where "birth" refers to the replication of a mutant infected cell; "death" is the death of a mutant infected cell; and "immigration" is the continual flow into the mutant population due to de novo mutations in the WT. We can read off these rates from Equation S3c substituting the values of $x^{\star}$ and $y_{w}^{\star}$ from Equations S4a and S4b to give,

$$
\begin{aligned}
B & =a_{r}(1-s) \\
D & =a_{r} \\
I & =a_{w} u y_{w}^{\star}
\end{aligned}
$$

The probability generating function (PGF) for the steady state distribution when $B<D$ is given by [90],

$$
\psi(z)=\left(\frac{B-D}{B z-D}\right)^{\frac{I}{B}}
$$

With the mean $\psi^{\prime}(1)=\frac{I}{D-B}$. The total survival probability is given by,

$$
P_{\text {pre }}=1-\sum_{n=0}^{\infty} \operatorname{Pr}(N=n) p_{\text {ext }}(0)^{n}=1-\psi\left(p_{\text {ext }}(0)\right)
$$

\section{Rescue}

The rate of appearance of rescue mutants is proportional to the level of residual infection of the WT and the mutation probability. This rate can be read off from Equation S4c,

$$
\nu(t)=u \frac{\beta_{w}(t) k}{c} x(t) y_{w}(t)
$$

Once a mutant is produced at time $t^{\prime}$, its establishment probability is given by Equation S11 calculated at $t=t^{\prime}$. Since we want to keep track of only those mutants that avoid extinction, the total rate of producing rescue mutants is,

$$
\nu_{\text {resc }}(t)=\nu(t) p_{\text {est }}(t)
$$

We model the number of rescue events that occur via a time inhomogeneous Poisson process [37] with the above rate and calculate probability that at least one rescue mutant establishes infection,

$$
P_{\text {resc }}=1-e^{-m_{1} p_{\text {est }}(0)-\int_{0}^{\infty}(1 / d) \nu(t / d) \mathrm{d} t}
$$

where $m_{1}=\left(1-\epsilon_{w}(t=0)\right) \frac{u y_{w}^{\star} a}{c}$ is a correction term that is needed because of the quasi-equilibrium approximation used for setting the free virus population proportional to the infected cells [37].

\section{Latency reactivation}

For mutants produced via latency reactivation, the establishment probability $p_{\text {est }}(t)$ is calculated as before by modeling this as a Birth-Death process with rates, $B(t)=b_{r}(t) x(t)$ and $D=a$. We approximate the target and WT infected cell dynamics by solving the following deterministic system,

$$
\begin{aligned}
\dot{x} & =\lambda-d x-b_{w}(t) x y_{w} \\
\dot{y}_{w} & =\gamma\left(1-\frac{u}{s}\right)+(1-u) b_{w}(t) x y_{w}-a y_{w}
\end{aligned}
$$

The rate at which resistant mutants are produced due to latency reactivation can be read off from Equation S6, 


$$
\nu(t)=\left(u b_{w} x(t) y_{w}(t)+\frac{\gamma u}{s}\right)
$$

In this case we also just keep track of those mutants that escape stochastic extinction and the total rate is given by,

$$
\nu_{\text {latency }}(t)=\nu(t) p_{\text {est }}(t)
$$

and the average probability of rescue per latency reactivation event is,

$$
P_{\text {latency }}=1-e^{-\left\langle\nu_{\text {latency }}(t)\right\rangle / \gamma}
$$

where, $\left\langle\nu_{\text {latency }}(t)\right\rangle$ is the rate averaged over one period of the drug cycle. The establishment of resistance due all the three sources was computed numerically using Mathematica 12.0 [91].

\section{Stochastic simulations of the birth-death approximation}

We checked the validity of the birth-death approximation used for all the three sources of resistance. For preexistence and rescue, we simulated the full model (Equation 1) stochastically using Gillespie's tau-leaping method [92]. The step size, $\Delta t$ was fixed to 0.001 time units. In this algorithm, the number of each type of event occurring in one time-step is drawn from a Poisson distribution with a mean $=$ event rate $\times \Delta t$. To obtain the establishment probability of a single pre-existing resistant mutant, we simulated the treatment phase multiple times $(10,000-100,000$ iterations). The initial conditions for the target cells and the WT infected cells were given by their pre-treatment equilibrium values with one resistant infected cell. We set the mutation rate to zero to isolate the establishment due to just the pre-existing mutant. The simulations were run long enough such that the mutant population had either established infection or gone extinct. The fraction of runs with surviving mutants estimates the establishment probability. For the rescue probability, we ran multiple stochastic simulations of the full model (10,000 iterations) with the same pre-treatment initial conditions for the target and WT infected cells. We set the initial resistant infected cell population to zero and included a non-zero mutation rate. The simulations were run long enough such that either a resistant infection had been established or the infection was cleared. The rescue probability corresponds to the fraction of runs in which the resistant infection was established.

To obtain the average probability of rescue per latency reactivation event, we stochastically simulated Equation S6 in three stages using Gillespie's tau-leaping method with the step size $\Delta t=0.001$. At the start of the simulation, the target cells are at their pre-infection equilibrium with no WT or resistant infected cells. In the first stage of the simulation we turn off the mutation rate and allow the WT population to settle into a form of an equilibrium where suppression by treatment is balanced by the activation of latent cells. In the second stage of the simulation we allow the reactivation of resistant latent cells and turn on the mutation rate for a period of $1 / \gamma$. Latency reactivation is a Poisson point process with a constant rate $\gamma$ where on average one reactivation event occurs during a period of $1 / \gamma$. This allows us to simulate the scenario of one reactivation event potentially leading to resistance. The probability of rescue (averaged over simulations) obtained in this manner is a good approximation of the probability per reactivation event as long as simulations where more than one reactivation event occurs do not contribute disproportionately to it. The probability of a resistant infection establishing due to $n$ reactivation events, each of which has a probability $p$ of causing infection, can be calculated by using the binomial distribution. In the limit of very small $p(<<1)$ however, this probability is well approximated by $n p$. As we are in this small $p$ regime, each reactivation event contributes equally to the probability and our approximation is valid. In the last stage, we turn off the mutation rate again and run the simulation for long enough such that the mutant has either gone extinct or established infection. Some of the probabilities involved are very small $O\left(10^{-5}\right)$ and in order to avoid running the large number of iterations, $\sim O\left(10^{7}\right)$ required to calculate these numbers (corresponding to weeks of computing time), we scaled up the mutation rate for the simulations and scaled down the final probability that was obtained. This is a reasonable step to take as when the probability is $<<1$, it is directly proportional to the mutation rate. For each set of parameter values, we allowed the second stage of the simulation to occur at 100 different time-points in the drug cycle and repeated this for 200 iterations each. The final probability is given by the fraction of runs (20,000 in total) where the mutant established infection. 
medRxiv preprint doi: https://doi.org/10.1101/2021.07.10.21260044; this version posted July 14, 2021. The copyright holder for this preprint

(which was not certified by peer review) is the author/funder, who has granted medRxiv a license to display the preprint in perpetuity.

It is made available under a CC-BY 4.0 International license .

All the simulations were implemented in Python 3.0 and run on Cannon, the Harvard University cluster.

\section{Existence of the establishment time-frame}

In order to demonstrate the dependence of pre-existence and rescue probabilities on a short initial timewindow after treatment onset, we computed the fraction of pre-existing mutants that have survived as a function of time after treatment onset (Figure S2). This can also be interpreted as the probability that the lineage of one pre-existing strain has avoided extinction by time $t, P\left(y_{r}(t) \neq 0 \mid y_{r}(0)=1\right)$. This probability is bounded by $P\left(y_{r}(0) \neq 0 \mid y_{r}(0)=1\right)=1$ and $P\left(y_{r}(\infty) \neq 0 \mid y_{r}(0)=1\right)=p_{\text {est }}$. If the establishment probability is reached in a finite amount of time, this implies that the lineage is guaranteed to establish infection if it survives until then. We find that this occurs rather quickly after treatment starts, and is inversely related to the infected cell decay rate $a$. Given the non-linear nature of the infection and treatment dynamics it is difficult to precisely measure the establishment time-frame. However, we don't consider this to be a limitation of our analysis as it is not needed to calculate the risks of resistance. The usefulness of the concept lies in helping understand and interpret the trends seen on varying drug dosing intervals on the risk of resistance.

\section{Models of non-adherence}

\section{Random adherence model}

In the random adherence model we assume that there is a constant probability of missing each dose apart from the first one which is always taken. The establishment and rescue probabilities were computed numerically using Mathematica 12.0 for the pharmacologically-inspired efficacy model.

\section{Clumped adherence model}

In the clumped adherence model we assume that apart from the first dose, each dose can be missed with a fixed probability $\left(p_{1}\right)$ but the probability of missing a dose is higher $\left(p_{2}>p_{1}\right)$ if the previous dose was missed. The convergent non-adherence probability $p$, can be computed by treating these two states $\left(\psi_{1}, \psi_{2}\right)$ as states of a stationary Markov Chain with the right stochastic transition matrix,

$$
T=\left(\begin{array}{ll}
1-p_{1} & p_{1} \\
1-p_{2} & p_{2}
\end{array}\right)
$$

Solving for the stationary distribution, $\psi T=\psi$ where $\psi=\left(\psi_{1} \psi_{2}\right)$, the average non-adherence probability is given by,

$$
p=p_{1} \psi_{1}+p_{2} \psi_{2}=\frac{p_{1}}{1-p_{2}+p_{1}}
$$

\section{Two-step mutations}

\section{Pre-existence}

The establishment probability of one pre-existing mutant is independent of the mutational pathway to resistance from the WT strain but the frequency distribution of the resistant strain prior to treatment changes. We generalize the PGF for the point mutants given by Equation S15 to two-step mutants using the method illustrated by Moreno-Gamez et al. [78] in their Supplementary Information.

An ODE model that can describe the dynamics of a system with a two-step mutant is given by [60,61], 


$$
\begin{aligned}
\dot{x} & =\lambda-d x-\frac{\beta_{w}(t) k}{c} x y_{w}-\frac{\beta_{m 1}(t) k}{c} x y_{m 1}-\frac{\beta_{m 2}(t) k}{c} x y_{m 2}-\frac{\beta_{r}(t) k}{c} x y_{r} \\
\dot{y}_{w} & =\left(1-u_{1}-u_{2}-u_{1} u_{2}\right) \frac{\beta_{w}(t) k}{c} x y_{w}-a y_{w} \\
\dot{y}_{m 1} & =u_{1} \frac{\beta_{w}(t) k}{c} x y_{w}+\left(1-u_{2}\right) \frac{\beta_{m 1}(t) k}{c} x y_{m 1}-a y_{m 1} \\
\dot{y}_{m 2} & =u_{2} \frac{\beta_{w}(t) k}{c} x y_{w}+\left(1-u_{1}\right) \frac{\beta_{m 2}(t) k}{c} x y_{m 2}-a y_{m 2} \\
\dot{y}_{r} & =\frac{\beta_{r}(t) k}{c} x y_{r}+u_{1} u_{2} \frac{\beta_{w}(t) k}{c} x y_{w}+u_{2} \frac{\beta_{m 1}(t) k}{c} x y_{m 1}+u_{1} \frac{\beta_{m 2}(t) k}{c} x y_{m 2}-a y_{r}
\end{aligned}
$$

where, $y_{m 1}$ and $y_{m 2}$ are two intermediate mutants a point mutation away from the WT with $u_{1}$ and $u_{2}$ as the two mutation rates and $y_{r}$ is the two-step mutant. The frequency distribution of the two-step mutant at the start of treatment can be approximated by considering the stochastic process determining the size of the single and double mutant populations $\left(y_{m 1}, y_{m 2}, y_{r}\right)$. We make the assumption that the target cell and WT populations are at their deterministic pre-treatment equilibrium values (Equations S4a and S4b). The selection coefficients are assumed to be larger than the mutation rates to get the following approximate rates,

$$
\begin{aligned}
y_{m 1} & \rightarrow y_{m 1}+1 \ldots \text { rate: } u_{1} a y_{w}^{\star}+\left(1-s_{1}\right) a y_{m 1} \\
y_{m 1} & \rightarrow y_{m 1}-1 \ldots \text { rate: } a y_{m 1} \\
y_{m 2} & \rightarrow y_{m 2}+1 \ldots \text { rate: } u_{2} a y_{w}^{\star}+\left(1-s_{2}\right) a y_{m 2} \\
y_{m 2} & \rightarrow y_{m 2}-1 \ldots \text { rate: } a y_{m 2} \\
y_{r} & \rightarrow y_{r}+1 \ldots \text { rate: } u_{1} u_{2} a y_{w}^{\star}+u_{1}\left(1-s_{2}\right) a y_{m 2}+u_{2}\left(1-s_{1}\right) a y_{m 1}+\left(1-s_{1}\right)\left(1-s_{2}\right) a y_{r} \\
y_{r} & \rightarrow y_{r}-1 \ldots \text { rate: } a y_{r}
\end{aligned}
$$

where $s_{1}, s_{2}$ are the two mutation costs. At this point we make a further approximation as this is no longer a B-D-I process and doesn't possess an analytic solution [78]. We make the approximation that if double mutants are frequent enough to affect treatment failure, then for realistic values of $u$ and $s$, single mutants should be frequent and well approximated by their constant equilibrium levels, $y_{m 1}^{\star}=\frac{u_{1}}{s_{1}} y_{w}^{\star}$ and $y_{m 2}^{\star}=\frac{u_{2}}{s_{2}} y_{w}^{\star}$. The stochastic system is modified to,

$$
\begin{aligned}
& y_{r} \rightarrow y_{r}+1 \ldots \text { rate: } u_{1} u_{2} a y_{w}^{\star}+u_{1}\left(1-s_{2}\right) a \frac{u_{2}}{s_{2}} y_{w}^{\star}+u_{2}\left(1-s_{1}\right) a \frac{u_{1}}{s_{1}} y_{w}^{\star} \\
& y_{r} \rightarrow y_{r}+1 \ldots \text { rate: }\left(1-s_{1}\right)\left(1-s_{2}\right) a y_{r} \\
& y_{r} \rightarrow y_{r}-1 \ldots \text { rate: } a y_{r}
\end{aligned}
$$

This is a modified B-D-I process with,

$$
\begin{aligned}
I & =u_{1} u_{2} a y_{w}^{\star}\left(\frac{1}{s_{1}}+\frac{1}{s_{2}}-1\right) \\
B & =\left(1-s_{1}\right)\left(1-s_{2}\right) a \\
D & =a
\end{aligned}
$$

and the PGF can be calculated by Equation S15.

\section{Rescue mutants}

As the establishment probability for one pre-existing mutant is unaffected by the mutational pathway to resistance, the only thing that changes in the case of the rescue mutants is their rate of production from the wild-type and intermediate strains. 
medRxiv preprint doi: https://doi.org/10.1101/2021.07.10.21260044; this version posted July 14, 2021. The copyright holder for this preprint (which was not certified by peer review) is the author/funder, who has granted medRxiv a license to display the preprint in perpetuity.

It is made available under a CC-BY 4.0 International license.

Equation S17 for the rate of resistant mutant production is modified to,

$$
\nu(t)=\frac{\beta(\epsilon) k}{c} x(t)\left(u_{1} u_{2} y_{w}(t)+u_{1}\left(1-s_{2}\right) y_{m 2}(t)+u_{2}\left(1-s_{1}\right) y_{m 1}(t)\right)
$$




\section{Supplementary figures}

A

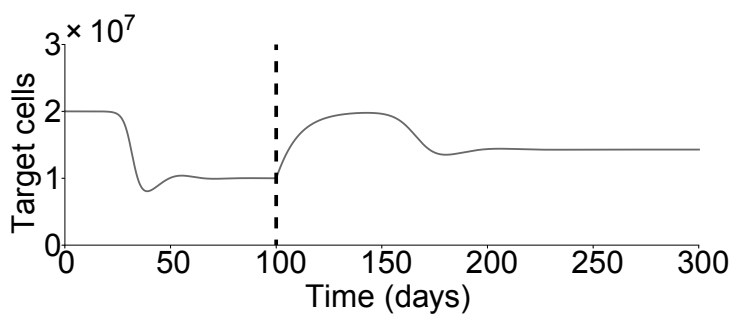

B

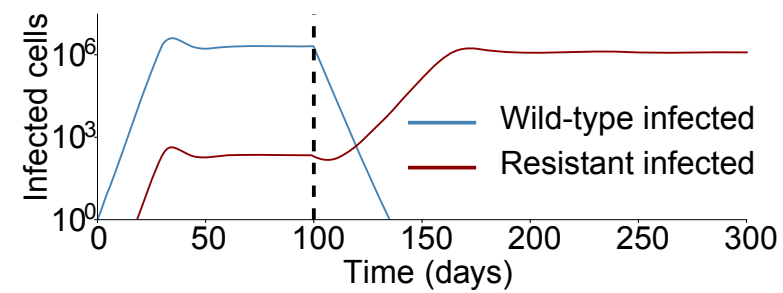

Figure S1: Example time-course of a chronic infection followed by treatment under the deterministic viral dynamics model. A) Target (uninfected) cells as a function of time. B) Time-course of cells infected by the wild-type (blue) and resistant (red) strain. Treatment is started $t=100$ days (vertical dotted line) after the onset of infection. Parameter values, $\lambda=2 \times 10^{6}$ cells/day, $d=0.1$ day $^{-1}, k=1000$ day $^{-1}, a=0.5$ day $^{-1}, c=10$ day $^{-1}, \beta^{0}=5 \times 10^{-10}$ day $^{-1}, u=3 \times 10^{-5}$ and $s=0.3$. Initial conditions, $x_{0}=\frac{\lambda}{d}, y_{s 0}=1$ and $y_{r 0}=0$. Before treatment, $\beta_{w}=\beta^{0}, \beta_{r}=\beta^{0}(1-s)$. After treatment, $\beta_{w}=\beta^{0}\left(1-\epsilon_{w}\right)$ with $\epsilon_{w}=0.9, A_{w}=0$ and $\epsilon_{r}(t)=0$.

A

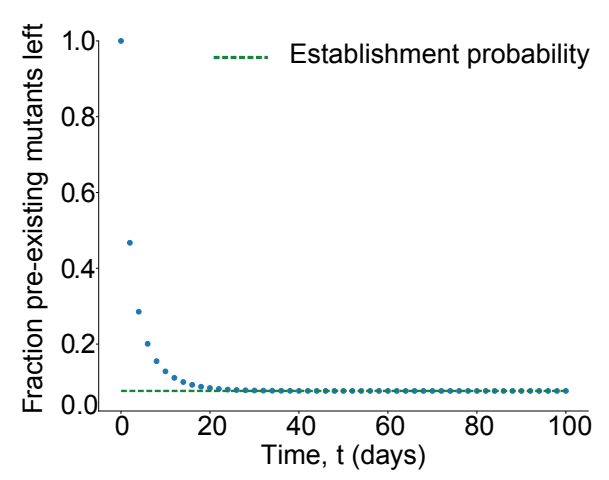

B

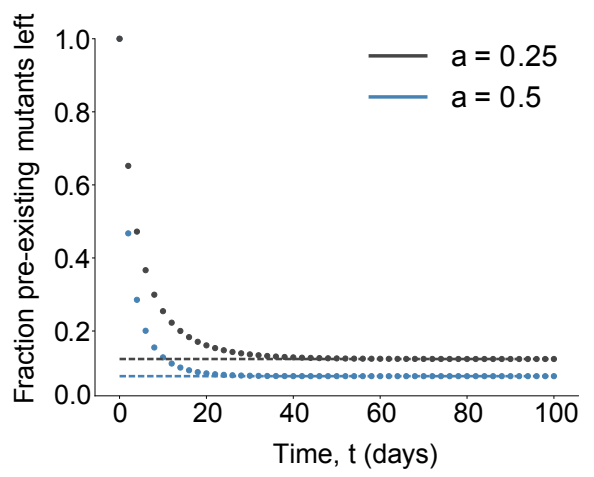

Figure S2: Fraction of pre-existing mutants left as a function of time post treatment onset. The dotted lines correspond to the establishment probability for a fully-resistant mutant in the presence of a pharmacologically-inspired drug efficacy model with dosing period $\mathrm{T}=1$ with $\mathrm{A}$ ) infected cell decay rate $a=0.5$ and $\mathrm{B}$ ) with two values of the infected cell decay rate $a=0.5$ (green) and $a=0.25$ (purple). The burst rate $k$ is adjusted according to the value of $a$ to ensure that $R_{0}$ for the two strains is the same in both cases. All other parameter values are the same as in Figure $2 \mathrm{~B}$ in the main text. 
Full resistance

\section{A}

\section{Constant drug efficacy}

\section{Sinusoidal drug efficacy}

C
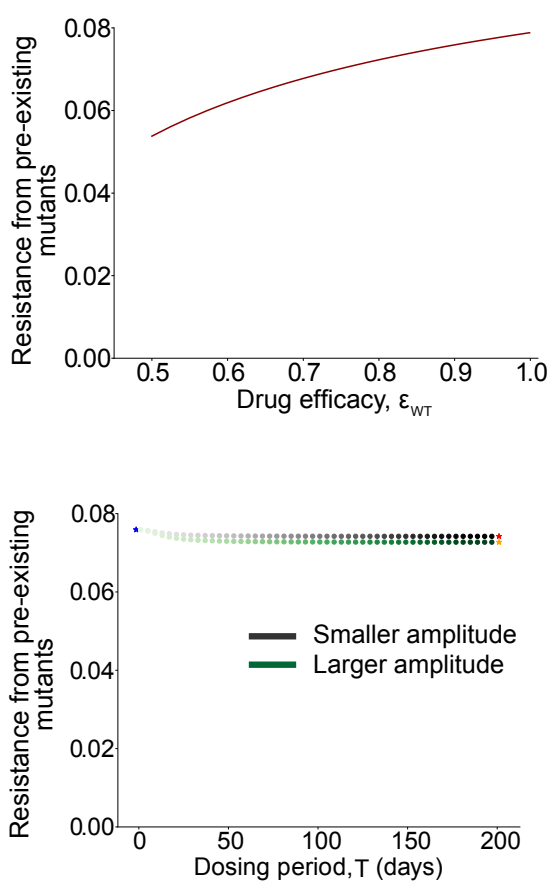

E

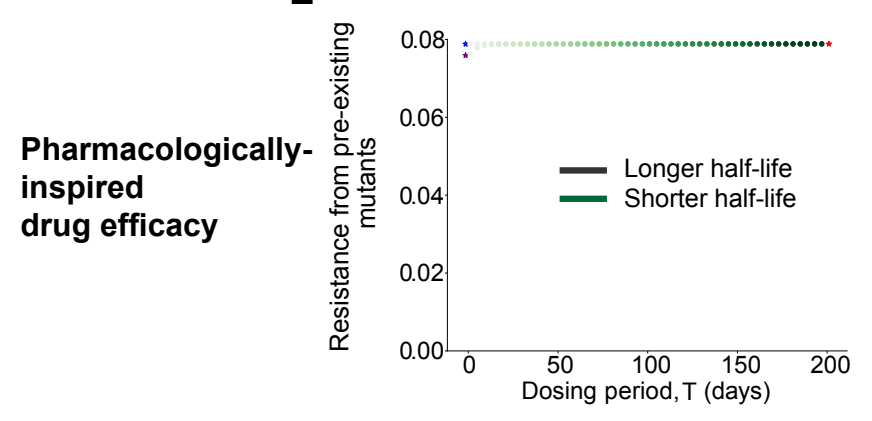

Partial resistance

B

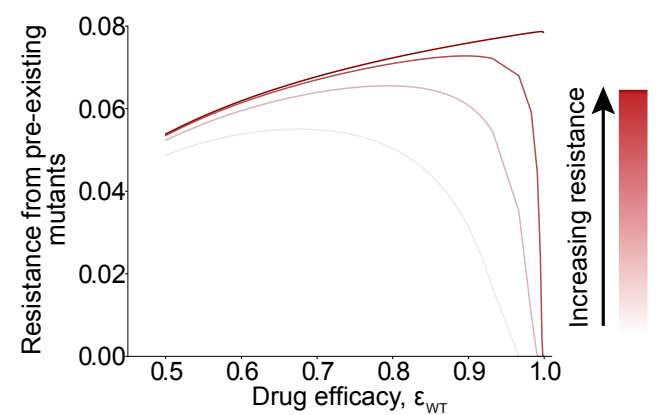

D

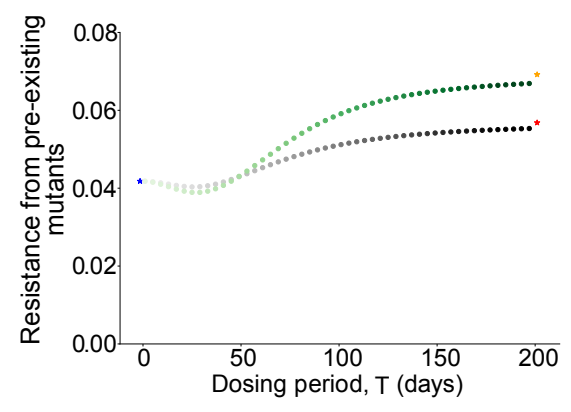

F

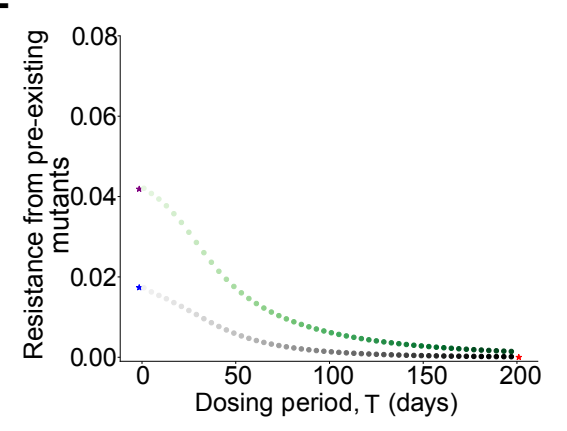

Figure S3: Effect of varying drug efficacy parameters on the establishment probability of pre-existing mutants. A)-B) Establishment probability as a function of constant drug efficacy for A) fully resistant $\left(\rho_{r}=\infty\right)$ B) partially resistant mutants $\left(\rho_{r}=1.5,1.7,2,4\right.$ with increasing darkness). C)-D) Effect of varying the amplitude keeping the average efficacy fixed in the sinusoidal efficacy model for a C) fully resistant and D) partially resistant mutant $\left(\rho_{r}=2\right)$. Parameter values for the green curves are the same as in Figure 2 in the main text with $A=0.05$ for the grey curves. The starred points correspond to the establishment probability for constant efficacies, $\epsilon_{\mathrm{avg}}=\langle\epsilon(t)\rangle$ (blue stars) and $\epsilon=\epsilon(t=0)$ (orange and red stars) E)-F) Effect of varying the half-life $t_{h}$ in the pharmacologically-inspired drug efficacy model keeping $C_{\max }$ fixed for a E) fully resistant and F) partially resistant mutant $\left(\rho_{r}=2\right)$. Parameter values for the green curves are the same as in Figure 2 in the main text with $t_{h} / T=5$ for the grey curves. The starred points correspond to the establishment probability for constant efficacies, $\epsilon_{\mathrm{avg}}=\langle\epsilon(t)\rangle$ (blue and purple stars) and $\epsilon=\epsilon(t=0)$ (red stars). 


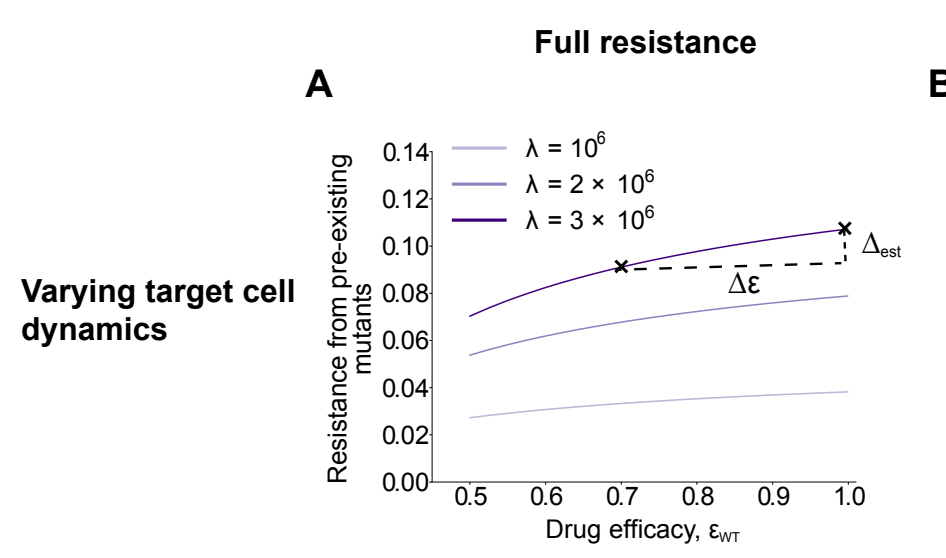

B

Partial resistance

C

\section{Varying infected cell dynamics}

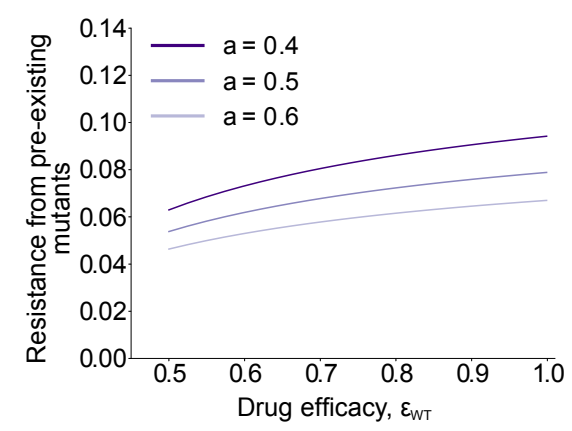

D
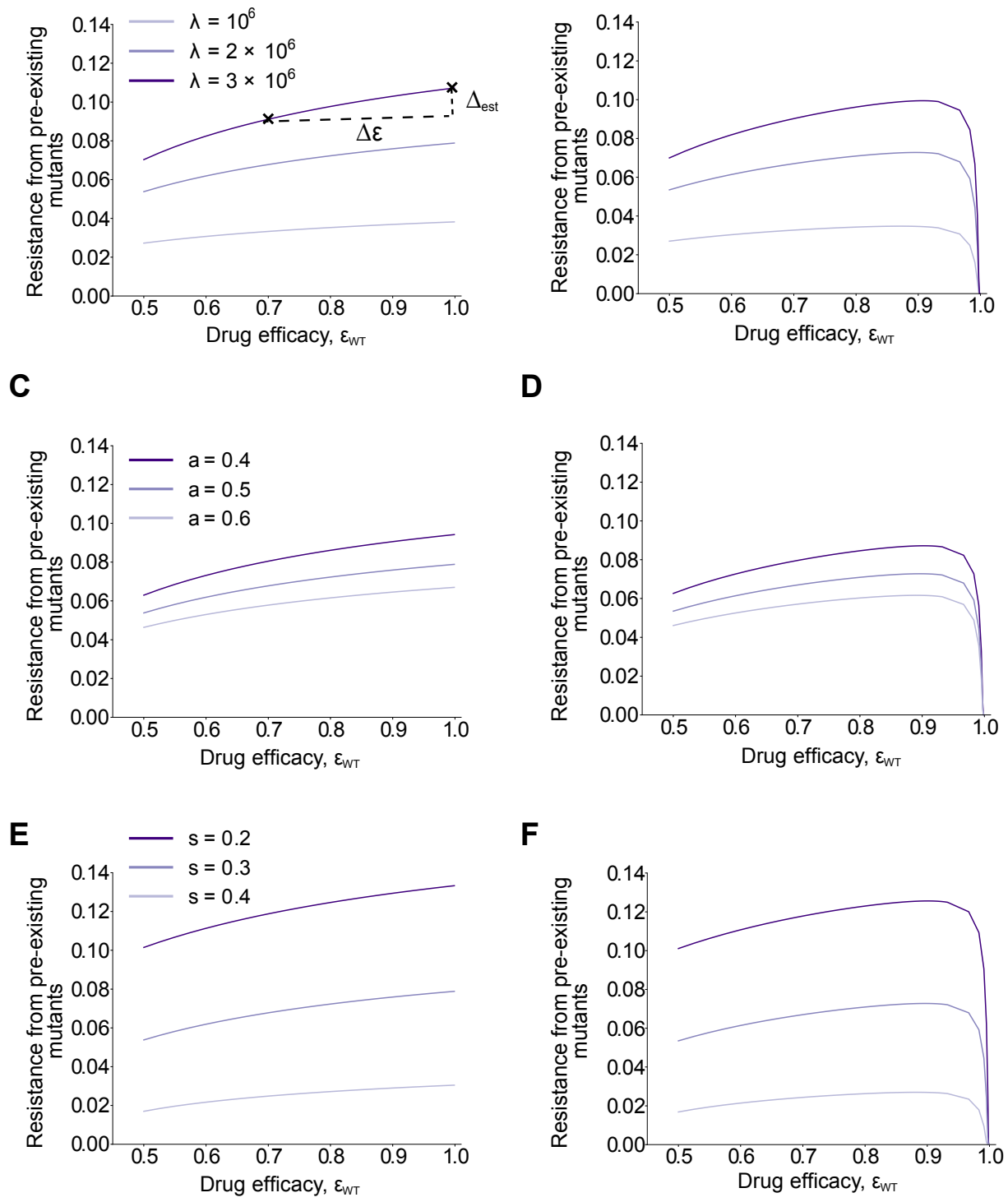

$\mathbf{F}$

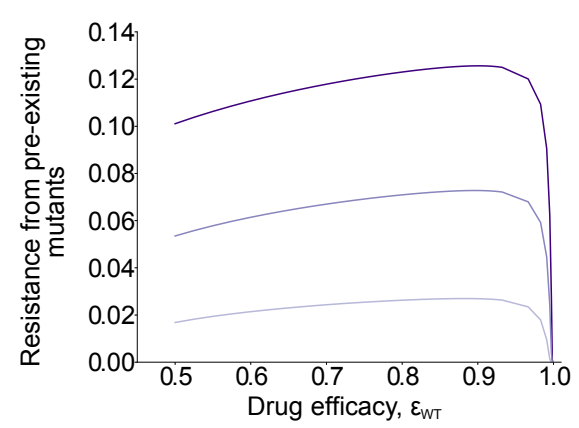

Figure S4: Effect of varying infection parameters on the establishment probability of pre-existing mutants in the presence of constant drug efficacy. A)-B) Establishment probability on varying the target (uninfected) cell production rate $\lambda$ for a A) fully and $\mathrm{B})$ partially resistant $\left(\rho_{r}=2\right)$ mutant. $\lambda$ increases as the color darkens with the death rate of uninfected cells $d$ increasing proportionally to keep $R_{0}$ for the wild-type fixed. Black marks in A) are for illustrative purposes to show the effect of increasing the dosing interval on the establishment probability. See Supplementary Discussion for more details. C)-D) Establishment probability for a C) fully and D) partially resistant $\left(\rho_{r}=2\right)$ mutant on varying the infected cell death rate $a$. $R_{0}$ for the wild-type is kept fixed by varying the infectivity parameter $b$ proportionally with $a$. E)-F) Effect of varying the cost of resistance $s$ for a $\mathrm{E})$ fully and $\mathrm{F})$ partially resistant $\left(\rho_{r}=2\right)$ mutant on the establishment probability. $s$ decreases as the color darkens. The efficacy on the x-axis corresponds to the wild-type drug efficacy. All other parameter values are the same as in Figure S1. 
medRxiv preprint doi: https://doi.org/10.1101/2021.07.10.21260044; this version posted July 14, 2021. The copyright holder for this preprint (which was not certified by peer review) is the author/funder, who has granted medRxiv a license to display the preprint in perpetuity.

\section{Constant drug efficacy}

\section{A}

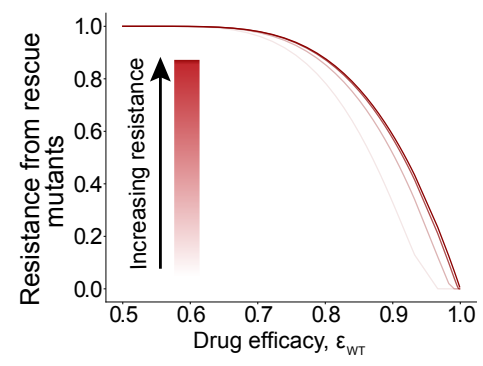

Sinusoidal drug efficacy

B

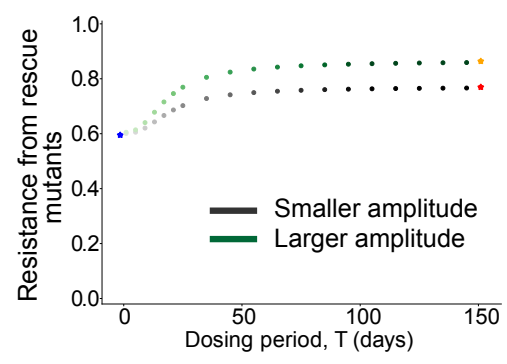

Pharmacologically-inspired drug efficacy

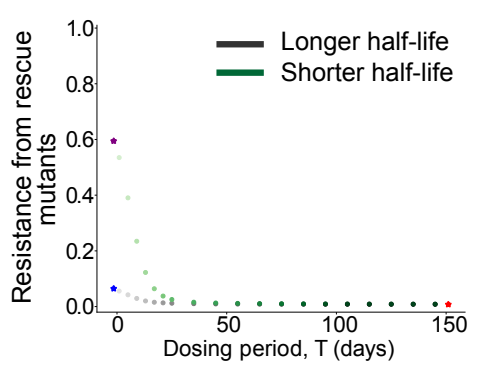

Figure S5: Effect of varying drug efficacy parameters on the rescue probability. A) Rescue probability as a function of constant efficacy for fully $\left(\rho_{r}=\infty\right)$ and partially resistant $\left(\rho_{r}=1.5,1.7,2,4\right.$ with increasing darkness $)$ mutants. B) Effect of varying the amplitude keeping the time-averaged drug efficacy fixed in the sinusoidal efficacy model for a fully resistant mutant. Parameter values for the green curves are the same as in Figure 2 in the main text with $A_{1}=0.05$ for the grey curves. The starred points correspond to the establishment probability for constant efficacies, $\epsilon_{\text {avg }}=\langle\epsilon(t)\rangle$ (blue stars) and $\epsilon=\epsilon(t=0)$ (orange and red stars) C) Effect of varying the half-life $t_{h}$ in the pharmacologically-inspired drug efficacy model keeping $C_{\text {max }}$ fixed for a fully resistant mutant. Parameter values for the green curves are the same as in Figure 2 in the main text with $t_{h} / T=5$ for the grey curves. The starred points correspond to the rescue probability for constant efficacies, $\epsilon_{\mathrm{avg}}=\langle\epsilon(t)\rangle(\mathrm{blue}$ and purple stars) and $\epsilon=\epsilon(t=0)$ (red star).

A

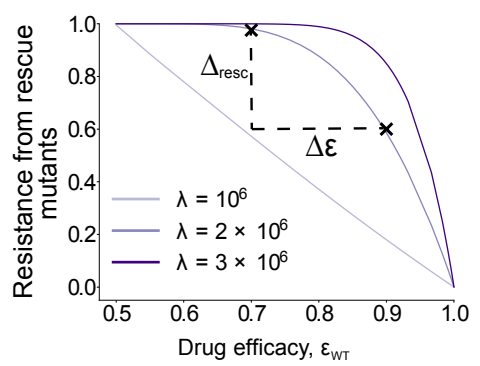

B

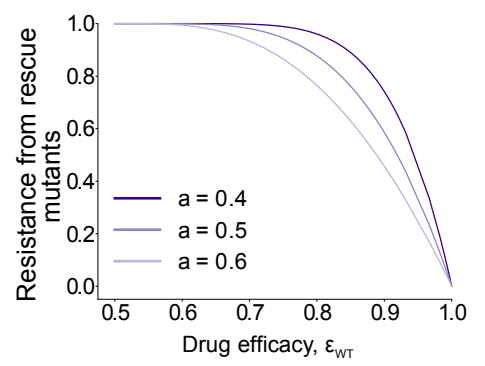

C

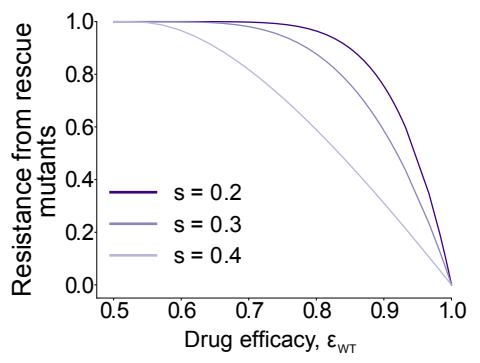

Figure S6: Effect of varying infection parameters on the rescue probability. A) Rescue probability on varying the target (uninfected) cell production rate $\lambda$ for a fully resistant mutant as a function of a constant drug efficacy. $\lambda$ increases as the color darkens, with the death rate of uninfected cells $d$ increasing proportionally to keep $R_{0}$ of the wild-type fixed. Black marks are for illustrative purposes to show the effect of increasing the dosing interval on the rescue probability. See Supplementary Discussion for more details. B) Effect of varying the infected cell death rate $a$ on the rescue probability for a fully resistant mutant as a function of a constant drug efficacy. a decreases as the color darkens. C) Rescue probability on varying the cost of mutation $s$ for a fully resistant mutant as a function of a constant drug efficacy. $s$ decreases as the color darkens. 
medRxiv preprint doi: https://doi.org/10.1101/2021.07.10.21260044; this version posted July 14, 2021. The copyright holder for this preprint (which was not certified by peer review) is the author/funder, who has granted medRxiv a license to display the preprint in perpetuity.

It is made available under a CC-BY 4.0 International license.

\section{Pharmacologically-inspired drug efficacy}

A

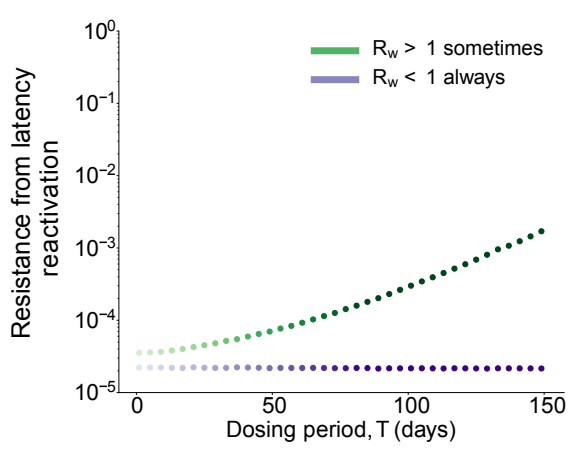

Sinusoidal drug efficacy

B

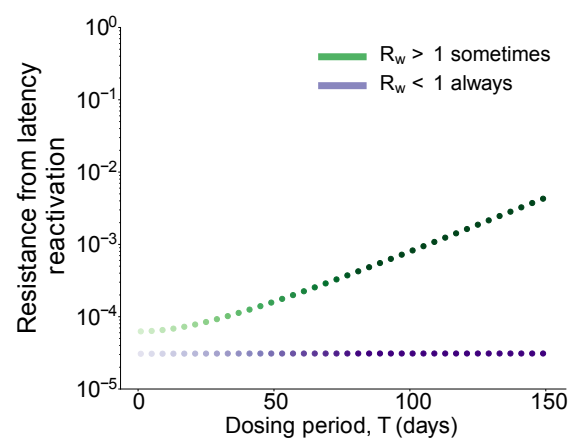

Figure S7: Effect of drug dosing intervals on the establishment of resistance due to latency reactivation. A) Average probability of rescue per latency reactivation event as a function of the dosing period for a partially resistant mutant under the pharmacologically-inspired drug efficacy model. The purple curve corresponds to a high drug efficacy such that $R_{w}<1$ during all points of the drug cycle. $R_{w}>1$ for $\sim 23 \%$ of each period for the low efficacy drug cycle given by the green curve. Parameter values used for the drug kinetics, $C_{\max }=20$ (purple curve), $C_{\max }=17$ (green curve), $I C_{50}=10, M=10$, $\frac{t_{h}}{T}=1, \rho_{w}=1, \rho_{r}=2$. B) Average probability of rescue per latency reactivation event as a function of the dosing period for a fully resistant mutant under the sinusoidal efficacy model. The purple curve corresponds to a high drug efficacy such that $R_{w}<1$ during all points of the drug cycle. $R_{w}>1$ for $\sim 30 \%$ of each period for the low efficacy drug cycle given by the green curve. Parameter values used for the drug kinetics, $\epsilon_{\mathrm{avg}, \mathrm{w}}=0.9, A_{w}=0.09$ (purple curve) and $\epsilon_{\mathrm{avg}, \mathrm{w}}=0.6, A_{w}=0.25$ (green curve) with $\epsilon_{r}(t)=0$ for both. A)-B) The colors go from light to dark with increasing periodicity keeping the time-averaged efficacy constant. 
medRxiv preprint doi: https://doi.org/10.1101/2021.07.10.21260044; this version posted July 14,2021 . The copyright holder for this preprint (which was not certified by peer review) is the author/funder, who has granted medRxiv a license to display the preprint in perpetuity.

It is made available under a CC-BY 4.0 International license.

\section{Full resistance}

A

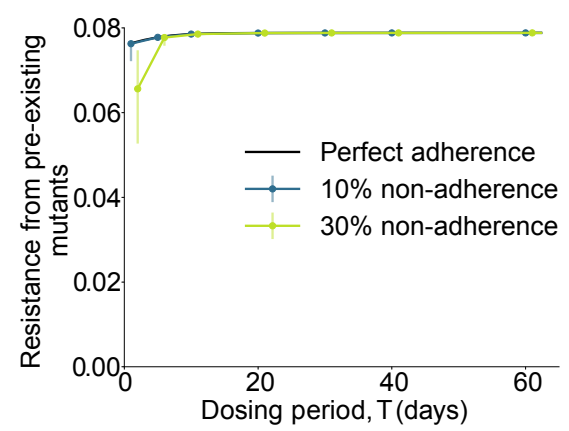

\section{Partial resistance}

C

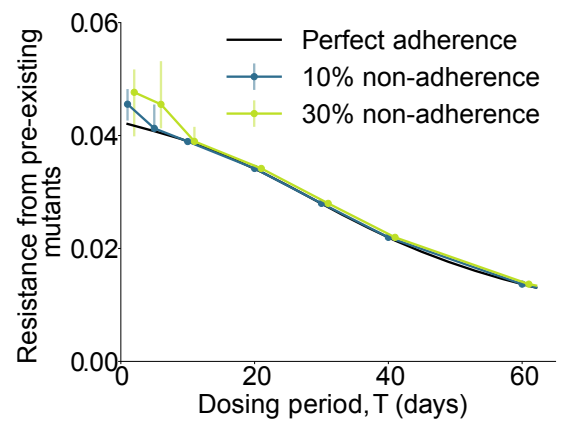

B
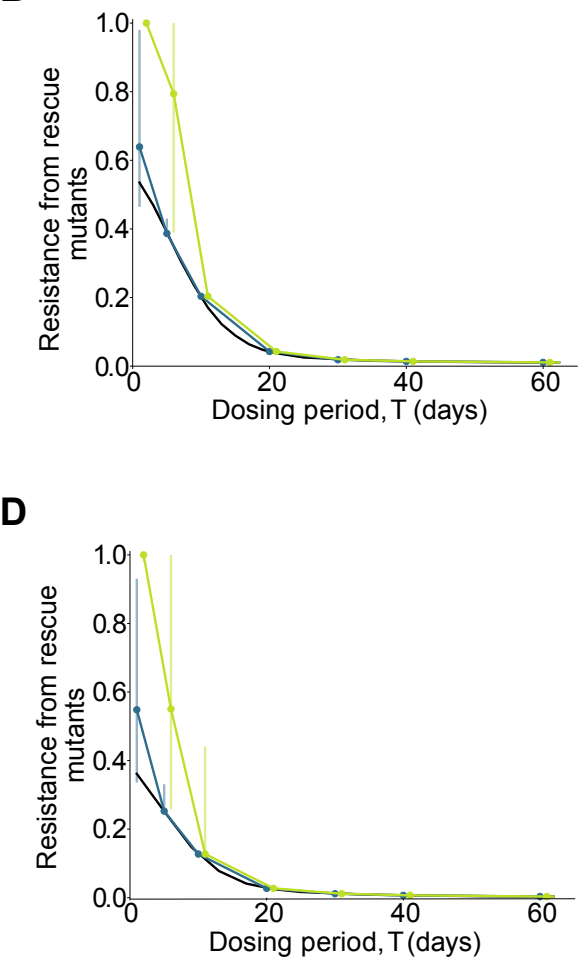

Figure S8: Effect of non-adherence on the establishment of resistance for different drug dosing intervals for a clumped adherence model. Establishment probability of A) fully $\left(\rho_{r}=\infty\right)$ and C) partially $\left(\rho_{r}=2\right)$ resistant pre-existing mutants. Probability that at least one B) fully $\left(\rho_{r}=\infty\right)$ and D) partially $\left(\rho_{r}=2\right)$ resistant rescue mutant is produced. These results are under the pharmacologically-inspired drug efficacy model with the solid black line corresponding to results for perfect adherence. Non-adherence is modeled using the clumped adherence model where the probability of missing a dose is higher if the previous dose was missed. Imperfect adherence results are medians of 100 iterations and the error bars correspond to the interquartile range. X-axis positions have been offset for ease of visualization. 


\section{Supplementary discussion}

\section{Role played by treatment parameters on the establishment probability for pre- existing mutants}

\section{Mutants fully resistant to treatment}

In the presence of a constant drug efficacy, the establishment probability for mutants fully resistant to treatment is higher as the drug efficacy increases (Figure S3A). Stronger wild-type (WT) suppression is beneficial to the mutant strain due to reduced competition for target cells.

When the drug efficacy is time varying, the establishment probability as a function of dosing intervals is constrained on both ends (very frequent and infrequent dosing) by the probabilities associated with the time-averaged drug efficacy and the initial drug efficacy (Figures S3C,E). Depending upon whether the initial efficacy is lower (higher) than the time-average, the establishment probability decreases (increases) as the dosing interval increases. The maximum possible effect of dosing intervals in this case is equal to the difference between the two constraining probabilities $\Delta_{\text {est }}=\left|P_{\text {est }}\left(\epsilon_{\text {avg }}\right)-P_{\text {est }}(\epsilon(t=0))\right|$.

Understanding how $\Delta_{\text {est }}$ depends upon the treatment parameters is straightforward in the sinusoidal model as we've parameterized it by the time-averaged drug efficacy $\epsilon_{\text {avg }}$ and the amplitude $A$.

$$
\epsilon(t)=\epsilon_{\mathrm{avg}}-A \cos \left(\frac{2 \pi t}{T}\right)
$$

The change in efficacy between these two extremes is given by, $\Delta \epsilon=\epsilon_{\text {avg }}-\left(\epsilon_{\text {avg }}-A\right)=A$. $\Delta_{\text {est }} \propto \Delta \epsilon$ and hence, increasing the amplitude increases the effect due to dosing intervals. We fix $\epsilon_{\text {avg }}$ and vary the amplitude in Figure S3C. The effect of drug kinetics is more pronounced when the amplitude is larger.

In the pharmacologically-inspired drug efficacy model,

$$
\epsilon(t)=1-\frac{1}{1+\left(\frac{C_{\max 2}{ }^{-t / t_{h}}}{\rho I C_{50}}\right)^{M}}
$$

Dependence of treatment parameters on $\Delta_{\text {est }}$ is more complicated in this case. Any parameter combination that increases $\Delta \epsilon$ corresponds to a larger $\Delta_{\text {est }}$. One way to see this is by varying the half-life $t_{h}$ (Figure S3E) keeping $\epsilon(t=0)$ fixed. The effect is more pronounced for drugs with a shorter half-life.

\section{Mutants partially resistant to treatment}

Treatment induces two opposing selection forces on a partially resistant strain - direct suppression of the strain by the drug and an indirect beneficial effect due to WT suppression that leads to more availability of target cells. Depending upon their relative intensity, a treatment with higher efficacy can increase or decrease the establishment probability for the resistant strain. To illustrate this, we plot the establishment probability as a function of constant drug efficacy for varying degree of resistance in Figure S3B. Due to the non-monotonic nature, increasing the drug efficacy doesn't always increase the establishment probability in this case. Depending upon the degree of resistance, the suppression of the resistant strain by treatment can overpower the benefit conferred to it by a strong WT suppression and lead to a lower establishment probability.

The establishment probability as a function of the dosing intervals is again constrained on both ends by the probabilities due to the time-averaged drug efficacy and the initial drug efficacy (Figures S3D,F).

For the sinusoidal drug efficacy model, the amplitude $A$ still controls the difference between the two limiting cases and a smaller amplitude corresponds to a smaller $\Delta_{\text {est }}$ (Figure S3D). The difference between the fully resistant case is that $\Delta_{\text {est }}$ is not the maximum possible difference due to dosing intervals anymore. This is on account of the more complicated trends for intermediate dosing intervals. Similarly, for the pharmacologically-inspired drug efficacy model, increasing $\Delta \epsilon$ increases $\Delta_{\text {est }}$ (Figure S3F). Shorter half-life of the drug leads to a larger effect of the drug dosing intervals on the establishment probability. 
medRxiv preprint doi: https://doi.org/10.1101/2021.07.10.21260044; this version posted July 14, 2021. The copyright holder for this preprint (which was not certified by peer review) is the author/funder, who has granted medRxiv a license to display the preprint in perpetuity. It is made available under a CC-BY 4.0 International license.

\section{Role played by treatment parameters on the rescue probability}

As mentioned in the main text, the rescue probability is always dominated by the residual rate of mutant production from the WT and hence, has a similar dependence on dosing intervals for both mutants fully and partially resistant to treatment. This is reflected in the rescue probability vs constant drug efficacy plot for varying degrees of resistance (Figure S5A). Treatment with higher efficacy is always worse for rescue mutants. The rescue probability as a function of the dosing interval is also constrained on both ends by the probabilities due to the time-averaged drug efficacy and the initial drug efficacy (Figures S5B,C). Depending upon whether the initial efficacy is higher (lower) than the average leads to a lower (higher) probability of rescue. Similar to the case of pre-existance we define, $\Delta_{\text {resc }}=\left|P_{\text {resc }}\left(\epsilon_{\text {avg }}\right)-P_{\text {resc }}(\epsilon(t=0))\right|$.

In the case of sinusoidal drug efficacy $\Delta_{\text {resc }} \propto A$ and a larger amplitude corresponds to a larger impact of dosing kinetics (Figure S5B). This is irrespective of whether the degree of resistance of the mutant strain. Similarly, for the pharmacologically-inspired drug efficacy model, larger $\Delta \epsilon$ corresponds to a larger $\Delta_{\text {resc }}$ (Figure S5C).

\section{Role played by the infection parameters on the establishment probability of pre- existing mutants}

The establishment probability of a pre-existing resistant mutant is dependent upon the infection parameters controlling the rate of target cell dynamics, infected cell dynamics and the cost of resistance. We plot the establishment probability as a function of a constant drug efficacy with varying target cell production rates $\lambda$ (Figures S4A and $\mathbf{S 4 B}$ ). $R_{0}$ of the WT is kept fixed (ratio $\lambda / d$ is fixed) in order to isolate the effects of just changing the timescale of the target cell dynamics. The establishment probability increases with faster target cell dynamics. The resistant strain can establish infection sooner due to faster target cell availability, lowering the chance of stochastic extinction. We also vary the infected cell death rate $a$ (Figures $\mathbf{S 4 C}$ and S4D) ( $R_{0}$ of the WT is kept fixed by varying the infectivity parameter $b$ proportionally to $a$ ) and the cost of resistance $s$ (Figures $\mathbf{S} 4 \mathbf{E}$ and $\mathbf{S} 4 \mathbf{F}$ ). Slower death rate and a lower cost of resistance reduces the chance of stochastic extinction and increases the establishment probability. In all of these cases, as $\Delta$ est $\propto \Delta \epsilon$, given an initial and a time-averaged drug efficacy (for example, black marks in Figure S4A), the relative difference between the establishment probabilities at these two extremes gives the extent to which drug dosing intervals affect the establishment probability. The effect of changing the drug dosing interval is therefore dependent on the local curvature of the establishment probability vs constant drug efficacy lines.

\section{Role played by the infection parameters on the rescue probability}

We also investigated the dependence of the rate of target cell dynamics, infected cell dynamics and the cost of resistance on the rescue probability in Figure S6. In all of these cases, we keep $R_{0}$ of the WT strain fixed in order to isolate the effect of just varying the parameters. Faster target cell dynamics (Figure S6A), slower death rate of infected cells (Figure S6B) and lower mutation costs (Figure S6C) lead to higher rates of rescue. As for pre-existence, given a time-averaged and an initial drug efficacy, the maximum possible effect of changing the drug dosing interval $\left(\Delta_{\text {resc }}\right)$ is dependent on the local curvature of the rescue probability vs constant drug efficacy lines (for example, black marks in Figure S6A). 